\title{
SHORTING, PARALLEL ADDITION AND FORM SUMS OF NONNEGATIVE SELFADJOINT LINEAR RELATIONS
}

\author{
YU.M. ARLINSKII
}

\begin{abstract}
We extend the operations of shorting and parallel addition from the cone of bounded nonnegative selfadjoint operators in a Hilbert space to the set of all nonnegative selfadjoint linear relations. New properties of these operations and connections with the Cayley transforms are established. It is shown that for a pair of nonnegative selfadjoint linear relations there exist, in general, two arithmetic-harmonic means. Applications of the arithmetic, harmonic, and arithmetic-harmonic means to the theory of nonnegative selfadjoint extensions of nonnegative symmetric linear relations are given.
\end{abstract}

\section{INTRODUCTION}

We will use the following notations: $\operatorname{dom} A, \operatorname{ran} A$, and $\operatorname{ker} A$ are the domain, the range, and the kernel of a linear operator/linear relation $A, \overline{\operatorname{ran}} A$ and clos $\mathcal{L}$ denote the closure of $\operatorname{ran} A$ and of the set $\mathcal{L}$, respectively. By s - lim we denote the strong limit of operators and $\mathrm{s}-\mathrm{R}-\mathrm{lim}$ means the strong resolvent limit of operators/linear relations [17, 33]. The Banach space of all bounded operators acting between Hilbert spaces $\mathcal{H}_{1}$ and $\mathcal{H}_{2}$ is denoted by $\mathbf{B}\left(\mathcal{H}_{1}, \mathcal{H}_{2}\right)$ and $\mathbf{B}(\mathcal{H}):=\mathbf{B}(\mathcal{H}, \mathcal{H})$. The cone of all bounded self-adjoint nonnegative operators in a complex Hilbert space $\mathcal{H}$ is denoted by $\mathbf{B}^{+}(\mathcal{H})$. If $A_{1}, A_{2}$ are two bounded selfadjoint operators in $\mathcal{H}$ and the difference $A_{1}-A_{2}$ belongs to $\mathbf{B}^{+}(\mathcal{H})$, then we write $A_{1} \geq A_{2}$. If $\mathcal{L}$ is a subspace (closed linear manifold) in $\mathcal{H}$, then $P_{\mathcal{L}}$ is the orthogonal

projection in $\mathcal{H}$ onto $\mathcal{L}$, and $\mathcal{L}^{\perp} \stackrel{\text { def }}{=} \mathcal{H} \ominus \mathcal{L}$. $\mathbb{N}$ is the set of natural numbers, $\mathbb{N}_{0}:=\mathbb{N} \cup\{0\}$, $\mathbb{C}$ is the field of complex numbers, $\mathbb{R}_{+}:=[0, \infty)$.

Let $\mathcal{H}$ be a complex Hilbert space and let $S \in \mathbf{B}^{+}(\mathcal{H})$. It was discovered by M.G. Kreיn [34] that for an arbitrary subspace $\mathcal{L}$ of $\mathcal{H}$ the set

$$
\Xi(S, \mathcal{L}):=\left\{\widetilde{S} \in \mathbf{B}^{+}(\mathcal{H}): \widetilde{S} \leq S, \operatorname{ran} \widetilde{S} \subseteq \mathcal{L}\right\}
$$

has a maximal element $S_{\mathcal{L}}$. The operation $\langle S, \mathcal{L}\rangle \mapsto S_{\mathcal{L}}$ was applied in 34 to the problem of a description contractive selfadjoint extensions of a non-densely defined Hermitian contraction. The following representations of $S_{\mathcal{L}}$ and associated quadratic form were established in [34:

$$
S_{\mathcal{L}}=S^{\frac{1}{2}} P_{\mathfrak{M}} S^{\frac{1}{2}},\left(S_{\mathcal{L}} f, f\right)=\inf _{\varphi \in \mathcal{L}^{\perp}}\{(S(f+\varphi), f+\varphi)\}, \quad f \in \mathcal{H},
$$

2010 Mathematics Subject Classification. 47A06, 47A64, 47A20.

Key words and phrases. Nonnegative selfadjoint linear relation, shorting, parallel addition, harmonic mean, arithmetic-harmonic mean, selfadjoint extension. 
where $P_{\mathfrak{M}}$ is the orthogonal projection in $\mathcal{H}$ onto the subspace $\mathfrak{M}=\left\{f \in \mathcal{H}: S^{\frac{1}{2}} f \in \mathcal{L}\right\}$. Besides 34

$$
\operatorname{ran} S_{\mathcal{L}}^{\frac{1}{2}}=\operatorname{ran} S^{\frac{1}{2}} \cap \mathcal{L}, S_{\mathcal{L}}=0 \Longleftrightarrow \operatorname{ran} S^{\frac{1}{2}} \cap \mathcal{L}=\{0\} .
$$

A bounded selfadjoint operator $S$ admits the block operator matrix representation w.r.t. the orthogonal decomposition $\mathcal{H}=\mathcal{L}^{\perp} \oplus \mathcal{L}$ :

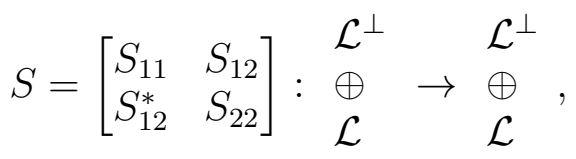

where $S_{11}^{*}=S_{11}, S_{22}^{*}=S_{22}$. It is well known (see e.g. [47]) that

$$
S \geq 0 \Longleftrightarrow\left\{\begin{array}{l}
S_{11} \geq 0, \operatorname{ran} S_{12} \subset \operatorname{ran} S_{11}^{\frac{1}{2}}, \\
S_{22}-\left(S_{11}^{\left[-\frac{1}{2}\right]} S_{12}\right)^{*}\left(S_{11}^{\left[-\frac{1}{2}\right]} S_{12}\right) \geq 0
\end{array}\right.
$$

and the operator $S_{\mathcal{L}}$ is given by the block matrix

$$
S_{\mathcal{L}}=\left[\begin{array}{lc}
0 & 0 \\
0 & S_{22}-\left(S_{11}^{\left[-\frac{1}{2}\right]} S_{12}\right)^{*}\left(S_{11}^{\left[-\frac{1}{2}\right]} S_{12}\right)
\end{array}\right]
$$

where $S_{11}^{\left[-\frac{1}{2}\right]}$ is the Moore-Penrose pseudo-inverse. If $S_{11}^{-1} \in \mathbf{B}\left(\mathcal{L}^{\perp}\right)$, then the operator $S_{22}-\left(S_{11}^{\left[-\frac{1}{2}\right]} S_{12}\right)^{*}\left(S_{11}^{\left[-\frac{1}{2}\right]} S_{12}\right)$ takes the form $S_{22}-S_{12}^{*} S_{11}^{-1} S_{12}$ and is said to be the $S c h u r$ complement of $S$ w.r.t. $\mathcal{L}$ [53]. From (1.5) it follows that

$$
S_{\mathcal{L}}=0 \Longleftrightarrow \operatorname{ran} S_{12} \subset \operatorname{ran} S_{11}^{\frac{1}{2}} \quad \text { and } \quad S_{22}=S_{12}^{*} S_{11}^{-1} S_{12} \text {. }
$$

The function $\mathbf{B}^{+}(\mathcal{H}) \ni S \mapsto S_{\mathcal{L}}$ has the following property [47]: in the strong convergence sense

$$
\mathbf{B}^{+}(\mathcal{H}) \supset\left\{S_{n}\right\} \searrow S_{\infty} \Longrightarrow\left(S_{n}\right)_{\mathcal{L}} \searrow\left(S_{\infty}\right)_{\mathcal{L}}
$$

For the case of a nonnegative $S$ acting on a finite-dimensional Hilbert space $\mathcal{H}$, the operator $S_{\mathcal{L}}$ in (1.5) was called by W.N. Anderson in [1] the shorted operator due to the its connection with electrical network theory. It was shown [1, Theorem 1] that if the shorted operator is defined by such a way, then it is the maximal element of the set $\Xi(S, \mathcal{L})$ defined in (1.1). Basic properties of $S_{\mathcal{L}}$ were studied in [1, 2, 3, 26, 34, 35, 39, 40, 42, 43, 47.

The parallel sum $B: G$ for $B, G \in \mathbf{B}^{+}(\mathcal{H})$ for the case of finite-dimensional $\mathcal{H}$ was defined in [2] as follows

$$
B: G=B(B+G)^{[-1]} G,
$$

where $(B+G)^{[-1]}$ is the Moore-Penrose pseudo-inverse. In the general case of $\operatorname{dim} \mathcal{H} \leq \infty$ the operator $B: G$ is of the form

$$
B: G=\left(B^{-1}+G^{-1}\right)^{-1},
$$

if both $B$ and $G$ have bounded inverses and if this is not the case, then

$$
\begin{aligned}
B: G=\mathrm{s}-\lim _{\varepsilon \downarrow 0}(B+\varepsilon I):(G+\varepsilon I)=\mathrm{s}-\lim _{\varepsilon \downarrow 0}\left((B+\varepsilon I)^{-1}\right. & \left.+(G+\varepsilon I)^{-1}\right)^{-1} \\
& =\mathrm{s}-\lim _{\varepsilon \downarrow 0} B(B+G+\varepsilon I)^{-1} G .
\end{aligned}
$$


In [26] the following important property of the parallel sum is established:

$$
\operatorname{ran}(B: G)^{\frac{1}{2}}=\operatorname{ran} B^{\frac{1}{2}} \cap \operatorname{ran} G^{\frac{1}{2}} .
$$

The quadratic form $((B: G) h, h)$ admits the representation

$$
((B: G) \varphi, \varphi)=\inf _{f, g \in \mathcal{H}}\{(B f, f)+(G g, g): \varphi=f+g\},
$$

see [3, 5, 26, 44]. It follows that $B: G \leq B$ and $B: G \leq G$. As is known [44], $B: G$ can be calculated as follows

$$
B: G=B-\left((B+G)^{[-1 / 2]} B\right)^{*}\left((B+G)^{[-1 / 2]} B\right),
$$

i.e., the parallel addition can be expressed [3] by means of the shorting:

$$
B: G=\left(\left[\begin{array}{cc}
B+G & B \\
B & B
\end{array}\right]\right)_{\{0\} \oplus \mathcal{H}}\lceil\{0\} \oplus \mathcal{H} .
$$

The latter representation leads to the following statement, see [44, Theorem 2.5]:

$$
\begin{aligned}
\mathbf{B}^{+}(\mathcal{H}) \supset\left\{B_{n}\right\},\left\{G_{n}\right\}, B_{n} \searrow B_{\infty}, G_{n} \searrow G_{\infty} & \text { in the strong convergence sense } \\
& \Longrightarrow \mathrm{s}-\lim _{n \rightarrow \infty}\left(B_{n}: G_{n}\right)=B_{\infty}: G_{\infty} .
\end{aligned}
$$

Another connections between shorting and parallel addition were established in [3] and are given by the following equalities

$$
\begin{gathered}
B_{\mathcal{L}}=\mathrm{s}-\lim _{t \uparrow+\infty}\left(B: t P_{\mathcal{L}}\right) \\
(B: G)_{\mathcal{L}}=B_{\mathcal{L}}: G=B: G_{\mathcal{L}}=B_{\mathcal{L}}: G_{\mathcal{L}}
\end{gathered}
$$

Various applications of shorting and parallel addition in complex analysis, operator theory, probability and statistics, numerical analysis are scattered in the literature. For matrices some of applications can be found in the books [38, 53]. Generalizations of the shorting and parallel additions to the cases of bounded operators, acting between two Hilbert spaces, on linear spaces, and on Krel̆n spaces, are given in [8, 19, 27, 37]. The operation of parallel addition has been extended to quadratic forms in [31, 32, 51, 52].

In this article the shorting and parallel addition are defined and studied for nonnegative selfadjoint linear relations (l.r. for short) and, in particular, for unbounded nonnegative selfadjoint operators. We define the shorting $A_{\mathcal{L}}$ similarly to Krel̆n's definition (1.1) for a bounded nonnegative selfadjoint operator. The parallel addition of two nonnegative selfadjoint l.r. we define as in (1.7), replacing the sum by the form sum. Recall that the form sum $A \dot{+} B$ of nonnegative selfadjoint l.r. $A$ and $B$ is the nonnegative selfadjoint l.r. associated with the closed quadratic form $\mathfrak{g}[\varphi]=\left\|A^{\frac{1}{2}} \varphi\right\|^{2}+\left\|B^{\frac{1}{2}} \varphi\right\|^{2}, \varphi \in \operatorname{dom} A^{\frac{1}{2}} \cap \operatorname{dom} B^{\frac{1}{2}}$ [25, 29, 33]. We show, see Theorem 5.4, that the inclusion $\operatorname{ran} A^{\frac{1}{2}}+\operatorname{ran} B^{\frac{1}{2}} \subseteq \operatorname{ran}(A \dot{+} B)^{\frac{1}{2}}$ holds. In the sequel the nonnegative selfadjoint l.r. $(A \dot{+} B) / 2$ will be called the arithmetic mean of $A$ and $B$. The harmonic mean $h(A, B)$ of $A$ and $B$ (in [36] the notation $A$ ! $B$ was used) is defined as follows

$$
h(A, B)=2(A: B)=\left(\frac{1}{2}\left(A^{-1} \dot{+} B^{-1}\right)\right)^{-1} .
$$

For $A, B \in \mathbf{B}^{+}(\mathcal{H})$ the inequality $h(A, B) \leq(A+B) / 2$ is valid [6].

For nonnegative selfadjoint l.r. we show that equalities (1.3), (1.8), (1.11) remain valid and some new properties of shorting and parallel additions we establish in Theorems 3.1 , 3.3, 4.2. 
There is a one-to-one correspondence between nonnegative selfadjoint l.r. $L$ and selfadjoint contractions $T$ given by the Cayley transform:

$$
\begin{aligned}
& L \mapsto \mathfrak{C}(L)=T:=-I+2(I+L)^{-1} \in[-I, I], \\
& {[-I, I] \ni T \mapsto \mathfrak{C}(T)=L:=-I+2(I+T)^{-1}=\{\{(I+T) f,(I-T) f\}: f \in \mathcal{H}\},} \\
& \mathfrak{C}(\mathfrak{C}(L))=L, \mathfrak{C}\left(L^{-1}\right)=-T=-\mathfrak{C}(L) .
\end{aligned}
$$

In Theorem 3.1 and Theorem 5.2 we establish the following equalities, connecting the Cayley transforms:

$$
\begin{aligned}
& \mathfrak{C}\left(A_{\mathcal{L}}\right)=I-(I-\mathfrak{C}(A))_{\mathcal{L}}, \\
& \mathfrak{C}\left(\frac{1}{2}(A \dot{+} B)\right)=h(I+\mathfrak{C}(A), I+\mathfrak{C}(B))-I, \\
& \mathfrak{C}(h(A, B))=I-h(I-\mathfrak{C}(A), I-\mathfrak{C}(B)),
\end{aligned}
$$

where $A$ and $B$ are nonnegative selfadjoint l.r. and $\mathcal{L}$ is a subspace. Using the above equalities and replacing the strong convergence by the strong resolvent convergence, in Proposition 3.2 and Corollary 5.3 we extend properties (1.6), (1.9), (1.10) to the set of all nonnegative selfadjoint l.r..

The mean $c_{0}(a, b):=\frac{a+b+2 a b}{2+a+b}$ of positive numbers $a$ and $b$ satisfies the inequalities

$$
\frac{2 a b}{a+b} \leq \frac{a+b+2 a b}{2+a+b} \leq \frac{a+b}{2}
$$

with " =" if and only if $a=b$. Using the Cayley transforms, the mean $c_{0}$ can be extended to arbitrary nonnegative selfadjoint l.r. $A$ and $B$ :

$$
c_{0}(A, B):=\mathfrak{C}\left(\frac{\mathfrak{C}(A)+\mathfrak{C}(B)}{2}\right) .
$$

We prove in Theorem 5.4 analogues of the above inequalities:

$$
h(A, B) \leq c_{0}(A, B) \leq \frac{A \dot{+} B}{2}
$$

with " = " if and only if $A=B$.

The geometric mean $A \# B$ for $A, B \in \mathbf{B}^{+}(\mathcal{H})$ can be defined as follows [6, 36, 45]:

$$
A \# B:=\left\{\begin{array}{l}
\max \left\{X \in \mathbf{B}^{+}(\mathcal{H}):|(X \varphi, \psi)| \leq\left\|A^{\frac{1}{2}} \varphi\right\|\left\|B^{\frac{1}{2}} \psi\right\| \varphi, \psi \in \mathcal{H}\right\}, \\
A^{\frac{1}{2}}\left(A^{-\frac{1}{2}} B A^{-\frac{1}{2}}\right)^{\frac{1}{2}} A^{\frac{1}{2}}, \text { if } A^{-1} \text { and } B^{-1} \text { are bounded }
\end{array}\right.
$$

For commuting $A$ and $B$ one has $A \# B=(A B)^{\frac{1}{2}}$. The following definition [4] of geometric mean as the arithmetic-harmonic mean is a straightforward generalization of this notion for positive numbers. Define

$$
A_{0}:=A, B_{0}:=B, A_{n}:=\frac{1}{2}\left(A_{n-1}+B_{n-1}\right), B_{n}:=h\left(A_{n-1}, B_{n-1}\right), n \in \mathbb{N} .
$$

Then $\left\{A_{n}\right\},\left\{B_{n}\right\}$ are non-increasing and non-decreasing sequences, respectively, and they have the common strong limit $g(A, B)$, which coincides with $A \# B$ and thus

$$
h(A, B) \leq A \# B \leq \frac{1}{2}(A+B) .
$$


In Section 6 we define similar sequences for nonnegative selfadjoint l.r. $A$ and $B$ and show that, in general, there is no a common strong resolvent limit, i.e., there are two arithmeticharmonic means $a h(A, B)$.

According to Kreŭn's results [34] and their generalizations in [7, 11, 18, 28, 29, 30] (see Section 7), the Cayley transform of the set of all nonnegative selfadjoint extensions of a nonnegative symmetric l.r. forms an operator interval. In Theorem 7.1 , in particular, we find parameters corresponding to the arithmetic and harmonic means of given two nonnegative selfadjoint extensions, in Proposition 7.2 we show that the pair of arithmetic-harmonic means $a h\left(\widetilde{S}_{1}, \widetilde{S}_{2}\right)$ for two extremal nonnegative selfadjoint extensions $\widetilde{S}_{1}, \widetilde{S}_{2}$ of a nonnegative symmetric operator or linear relation consists of $\frac{1}{2}\left(\widetilde{S}_{1}+\widetilde{S}_{2}\right)$ and $h\left(\widetilde{S}_{1}, \widetilde{S}_{2}\right)$. In Proposition 7.3 we show for differential operators in $\mathcal{L}_{2}\left(\mathbb{R}_{+}\right)$

$$
\left\{\begin{array}{l}
L_{c} f=-\frac{d^{2} f}{d x^{2}} \\
\operatorname{dom} L_{c}=\left\{f \in W_{2}^{2}\left(\mathbb{R}_{+}\right), f^{\prime}(0)=c f(0)\right\}, c \in[0,+\infty], W_{2}^{2}\left(\mathbb{R}_{+}\right) \text {is the Sobolev space }
\end{array}\right.
$$

the validity of the equalities

$$
\begin{aligned}
& \frac{1}{2}\left(L_{c} \dot{+} L_{d}\right)=L_{\frac{1}{2}(c+d)}, h\left(L_{c}, L_{d}\right)=L_{\frac{2 c d}{c+d}}, a h\left(L_{c}, L_{d}\right)=L_{\sqrt{c d}}, \\
& \frac{1}{2}\left(L_{0} \dot{+} L_{\infty}\right)=L_{\infty}, h\left(L_{0}, L_{\infty}\right)=L_{0}, a h\left(L_{\infty}, L_{0}\right)=\left\langle L_{\infty}, L_{0}\right\rangle .
\end{aligned}
$$

In the case of a nonnegative symmetric l.r. having one-dimensional resolvent difference of its Friedrichs and Kreln extensions, we calculate in Theorem 7.4 parameters corresponding to the resolvents of the arithmetic-harmonic means of any two nonnegative selfadjoint extensions.

\section{Nonnegative SELFADJoint LineAR RELATiOnS}

Let $\mathcal{H}$ be a Hilbert space and let

$$
J_{\mathcal{H}}:\left\{f, f^{\prime}\right\} \mapsto\left\{i f^{\prime},-i f\right\}, f, f^{\prime} \in \mathcal{H} .
$$

Then $J_{\mathcal{H}}$ is selfadjoint and unitary operator in the Hilbert space $\mathcal{H}^{2}=\mathcal{H} \oplus \mathcal{H}$. Let $A$ be a l.r. in $\mathcal{H}[9]$, i.e., $A$ is a closed linear manifold (a subspace) in the Hilbert space $\mathcal{H}^{2}$. Recall that the l.r. $A^{*}:=\mathcal{H}^{2} \ominus J_{\mathcal{H}} A$ is called the adjoint to $A$. If $A=\left\{\left\{\varphi, \varphi^{\prime}\right\}\right\}$, then, clearly,

$$
A^{*}=\left\{\left\{g, g^{\prime}\right\}:\left(\varphi^{\prime}, g\right)=\left(\varphi, g^{\prime}\right) \forall\left\{\varphi, \varphi^{\prime}\right\} \in A\right\} .
$$

A l.r. $A$ is called selfadjoint if $A^{*}=A \Longleftrightarrow A=\mathcal{H}^{2} \ominus J_{\mathcal{H}} A$. The domain, range, kernel and multi-valued part of a l.r. $A$ are defined as follows:

$$
\begin{aligned}
& \operatorname{dom} A=\left\{f \in \mathcal{H}:\left\{f, f^{\prime}\right\} \in A\right\}, \operatorname{ran} A=\left\{f^{\prime} \in \mathcal{H}:\left\{f, f^{\prime}\right\} \in A\right\}, \\
& \operatorname{ker} A=\{f \in \mathcal{H}:\{f, 0\} \in A\}, \operatorname{mul} A=\left\{f^{\prime} \in \mathcal{H}:\left\{0, f^{\prime}\right\} \in A\right\} .
\end{aligned}
$$

The l.r. $A^{-1}:=\left\{\left\{f^{\prime}, f\right\}:\left\{f, f^{\prime}\right\} \in A\right\}$ is called the inverse to $A$. Clearly

$$
\operatorname{dom} A^{-1}=\operatorname{ran} A, \operatorname{ran} A^{-1}=\operatorname{dom} A, \operatorname{ker} A^{-1}=\operatorname{mul} A, \operatorname{mul} A^{-1}=\operatorname{ker} A \text {. }
$$

Recall that a symmetric (selfadjoint) l.r. $A$ admits the orthogonal decomposition $A=$ $\operatorname{Graph}\left(A_{o}\right) \bigoplus\{\{0\} \oplus \operatorname{mul} A\}$, where $A_{o}$ is the symmetric (selfadjoint) operator part acting on $\overline{\operatorname{dom}} A(\overline{\operatorname{dom}} A=\mathcal{H} \ominus \operatorname{mul} A$ when $A$ is selfadjoint $)$.

The resolvent $(A-\lambda I)^{-1}$ of a selfadjoint l.r. $A$ is defined for all regular points $\rho\left(A_{o}\right)$ of the operator part $A_{o}$ and takes the form

$$
(A-\lambda I)^{-1} f=\left(A_{o}-\lambda I\right)^{-1} P_{A} f, f \in \mathcal{H},
$$

where $P_{A}$ is the orthogonal projection onto $\overline{\operatorname{dom}} A$. We will identify $\rho(A)$ with $\rho\left(A_{o}\right)$. 
If $\mathcal{L}$ is a subspace of $\mathcal{H}$, then $P_{\mathcal{L}}(A-\lambda I)^{-1}\lceil\mathcal{L}$ is called the compressed resolvent of $A$. The holomorphic family $\mathcal{M}_{A, \mathcal{L}}$ of l.r.

$$
\mathcal{M}_{A, \mathcal{L}}(\lambda):=-\left(P_{\mathcal{L}}(A-\lambda I)^{-1} \uparrow \mathcal{L}\right)^{-1}-\lambda I_{\mathcal{L}}, \lambda \in \rho\left(A_{o}\right)
$$

is a Nevanlinna function or a Nevanlinna family [21, 22]. Note that

$$
\mathcal{M}_{A^{-1}, \mathcal{L}}(\lambda)=\left(\mathcal{M}_{A, \mathcal{L}}\left(\lambda^{-1}\right)\right)^{-1}, \lambda \in \rho(A) \backslash\{0\} .
$$

If a bounded selfadjoint operator $A$ in the Hilbert space $\mathcal{H}=\mathcal{L}^{\perp} \oplus \mathcal{L}$ is given by the block operator matrix

then $\mathbf{B}(\mathcal{L})$-valued function

$$
A=\left[\begin{array}{ll}
A_{11} & A_{12} \\
A_{12}^{*} & A_{22}
\end{array}\right], A_{11}=A_{11}^{*}, A_{22}=A_{22}^{*},
$$

$$
\mathcal{M}_{A, \mathcal{L}}(\lambda):=-A_{22}+A_{12}^{*}\left(A_{11}-\lambda I_{\mathcal{L}^{\perp}}\right)^{-1} A_{12}, \quad \lambda \in \rho\left(A_{11}\right)
$$

is a Nevanlinna function and

$$
P_{\mathcal{L}}\left(A-\lambda I_{\mathcal{L}}\right)^{-1}\left\lceil\mathcal{L}=-\left(\mathcal{M}_{A, \mathcal{L}}(\lambda)+\lambda I_{\mathcal{L}}\right)^{-1}, \lambda \in \rho(A) \cap \rho\left(A_{11}\right) .\right.
$$

In the sequel the notation $A\lceil\mathcal{L}$ is used for the l.r.

$$
A\left\lceil\mathcal{L}:=\left\{\left\{f, f^{\prime}\right\}: f \in \operatorname{dom} A \cap \mathcal{L},\left\{f, f^{\prime}\right\} \in A\right\} .\right.
$$

It is established in [50] that if $A$ is a selfadjoint unbounded linear operator and $\mathcal{L}$ is a subspace with finite codimension, then the operator $P_{\mathcal{L}} A\lceil\mathcal{L}$ is selfadjoint in the space $\mathcal{L}$. In [41] has been proved that the compression $P_{\mathcal{L}} A\lceil\mathcal{L}$ of a maximal dissipative operator $A$ is maximal dissipative in $\mathcal{L}$. Further in [16] similar assertions were obtained for selfadjoint and maximal dissipative l.r..

Let $A=\left\{\left\{f, f^{\prime}\right\}\right\}$ be a symmetric l.r. in the Hilbert space $\mathcal{H}$. Then for any $\left\{f, f^{\prime}\right\} \in A$ one has the equality [46]

$$
\left(f^{\prime}, f\right)=\left(A_{o} f, f\right)
$$

A 1.r. $A$ is called nonnegative (we will write $A \geq 0$ ) if $\left(f^{\prime}, f\right) \geq 0$ for all $\left\{f, f^{\prime}\right\} \in A$.

If $A$ is a nonnegative selfadjoint l.r. then the square root $A^{\frac{1}{2}}$ is defined as follows

$$
\left.A^{\frac{1}{2}}=\operatorname{Graph}\left(A_{o}^{\frac{1}{2}}\right) \bigoplus\{\{0\} \oplus \operatorname{mul} A\}\right\}, \operatorname{ran} A^{\frac{1}{2}}=\operatorname{ran} A_{o}^{\frac{1}{2}} \oplus \operatorname{mul} A
$$

Hence

$$
A^{-\frac{1}{2}}=\left\{\left\{A_{o}^{\frac{1}{2}} f, f\right\}, f \in \operatorname{dom} A_{o}^{\frac{1}{2}}\right\} \bigoplus\{\{\operatorname{mul} A \oplus\{0\}\} .
$$

Let $\mathfrak{a}=\mathfrak{a}[\cdot, \cdot]$ be a nonnegative sesquilinear form in the Hilbert space $\mathfrak{H}$ with domain dom $\mathfrak{a}$ and let $\mathfrak{a}[\varphi]:=\mathfrak{a}[\varphi, \varphi], \varphi \in \operatorname{dom} \mathfrak{a}$. The form $\mathfrak{a}$ is closed [33] if

$$
\left\{\begin{array}{rl}
\lim _{n \rightarrow \infty} \varphi_{n} & =\varphi, \quad \lim _{n, m \rightarrow \infty} \mathfrak{a}\left[\varphi_{n}-\varphi_{m}\right]=0, \\
\left\{\varphi_{n}\right\} & \subset \operatorname{dom} \mathfrak{a}
\end{array} \Longrightarrow \varphi \in \operatorname{dom} \mathfrak{a}, \lim _{n \rightarrow \infty} \mathfrak{a}\left[\varphi_{n}-\varphi\right]=0 .\right.
$$

The form $\mathfrak{a}$ is closable [33] if

$$
\left\{\begin{array}{rl}
\lim _{n \rightarrow \infty} \varphi_{n} & =0, \quad \lim _{n, m \rightarrow \infty} \mathfrak{a}\left[\varphi_{n}-\varphi_{m}\right]=0, \\
\left\{\varphi_{n}\right\} & \subset \operatorname{dom} \mathfrak{a}
\end{array} \Longrightarrow \lim _{n \rightarrow \infty} \mathfrak{a}\left[\varphi_{n}\right]=0 .\right.
$$

The form $\mathfrak{a}$ is closable if and only if it has a closed extension, and in this case the closure of the form is the smallest closed extension of $\mathfrak{a}$. The inequality $\mathfrak{a}_{1} \geq \mathfrak{a}_{2}$ for semi-bounded forms $\mathfrak{a}_{1}$ and $\mathfrak{a}_{2}$ is defined by

$$
\operatorname{dom} \mathfrak{a}_{1} \subseteq \operatorname{dom} \mathfrak{a}_{2}, \quad \mathfrak{a}_{1}[\varphi] \geq \mathfrak{a}_{2}[\varphi], \quad \varphi \in \operatorname{dom} \mathfrak{a}_{1} .
$$


In particular, $\mathfrak{a}_{1} \subset \mathfrak{a}_{2}$ implies $\mathfrak{a}_{1} \geq \mathfrak{a}_{2}$. If the forms $\mathfrak{a}_{1}$ and $\mathfrak{a}_{2}$ are closable, the inequality $\mathfrak{a}_{1} \geq \mathfrak{a}_{2}$ is preserved by their closures.

If $A=\left\{\left\{f, f^{\prime}\right\}\right\}$ is a nonnegative symmetric l.r., then the form

$$
\mathfrak{a}[f]=\left(f^{\prime}, f\right),\left\{f, f^{\prime}\right\} \in A, f \in \operatorname{dom} A=\operatorname{dom} A_{o}
$$

is closable [33, 46]. There is a one-to-one correspondence between all closed nonnegative forms $\mathfrak{a}$ and all nonnegative selfadjoint l.r. $A$ in $\mathfrak{H}$ (the first representation theorem [33]), see [33, 46], via $\operatorname{dom} A \subset \operatorname{dom} \mathfrak{a}$ and

$$
\mathfrak{a}[f, \psi]=\left(A_{o} f, \psi\right), \quad f \in \operatorname{dom} A, \quad \psi \in \operatorname{dom} \mathfrak{a} .
$$

In what follows the closed form associated with $A$ is denoted by $A[\cdot, \cdot]$ and its domain by $\mathcal{D}[A]$. By the second representation theorem

$$
A[\varphi, \psi]=\left(A_{o}^{\frac{1}{2}} \varphi, A_{o}^{\frac{1}{2}} \psi\right), \quad \varphi, \psi \in \mathcal{D}[A]=\operatorname{dom} A_{o}^{\frac{1}{2}} .
$$

The formulas (2.5), (2.6) are analogs of Kato's representation theorems for, in general, nondensely defined closed semi-bounded forms in [33, Section VI]; see e.g. [46, 14, 30].

If $A$ is a nonnegative selfadjoint l.r. and if $T=\mathfrak{C}(A)$ is its Cayley transform, then [12]

$$
\left\{\begin{array}{l}
\mathcal{D}[A]=\operatorname{ran}(I+T)^{\frac{1}{2}} \\
\left.A[u, v]=-(u, v)+2(I+T)^{\left[-\frac{1}{2}\right]} u,(I+T)^{\left[-\frac{1}{2}\right]} v\right), u, v \in \mathcal{D}[A],
\end{array}\right.
$$

where $(I+T)^{\left[-\frac{1}{2}\right]}$ is the Moore-Penrose pseudo-inverse. Replacing $A$ by $A^{-1}$ in (2.7), we conclude that $\operatorname{ran} A^{\frac{1}{2}}=\operatorname{ran}(I-T)^{\frac{1}{2}}$.

Given a sequence $\left\{A_{n}\right\}$ of nonnegative selfadjoint l.r., we say that $\left\{A_{n}\right\}$ converges in the strong resolvent sense [33] to a nonnegative selfadjoint l.r. $A$

$\left(\mathrm{s}-\mathrm{R}-\lim _{n \rightarrow \infty} A_{n}=A\right)$ if the sequence of the resolvent $\left\{\left(A_{n}+I\right)^{-1}\right\}$ converges in the strong sense to the resolvent $(A+I)^{-1}\left(\mathrm{~s}-\lim _{n \rightarrow \infty}\left(A_{n}+I\right)^{-1}=(A+I)^{-1}\right)$.

Let $A_{1}$ and $A_{2}$ be nonnegative selfadjoint l.r. in $\mathcal{H}$, then $A_{1}$ and $A_{2}$ are said to satisfy the inequality $A_{1} \geq A_{2}$ if

$$
\operatorname{dom} A_{1, o}^{\frac{1}{2}} \subset \operatorname{dom} A_{2, o}^{\frac{1}{2}} \text { and }\left\|A_{1, o}^{\frac{1}{2}} \varphi\right\| \geq\left\|A_{2, o}^{\frac{1}{2}} \varphi\right\|, \quad \varphi \in \operatorname{dom} A_{1, o}^{\frac{1}{2}} .
$$

This means that the closed nonnegative forms $A_{1}[\cdot, \cdot]$ and $A_{2}[\cdot, \cdot]$ associated with $A_{1}$ and $A_{2}$ satisfy the inequality $A_{1}[\varphi] \geq A_{2}[\varphi]$ for all $\varphi \in D\left[A_{1}\right]$; see (2.4), (2.6). Finally we note that the inequalities $A_{1} \geq A_{2} \geq 0$ are equivalent to the inequality $A_{1}^{-1} \leq A_{2}^{-1}$, see [29]. Besides,

$$
A_{1} \geq A_{2} \geq 0 \Longleftrightarrow-I \leq \mathfrak{C}\left(A_{1}\right) \leq \mathfrak{C}\left(A_{2}\right) \leq I .
$$

Let $A$ be a nonnegative selfadjoint l.r. in $\mathcal{H}$. Then the resolvent $(A+x I)^{-1}$ is a bounded nonnegative selfadjoint operator for an arbitrary positive number $x$. If $0 \leq x_{1} \leq x_{2}$, then, clearly, $A^{-1} \geq\left(A+x_{1} I\right)^{-1} \geq\left(A+x_{2} I\right)^{-1}$ and

$$
\begin{aligned}
& \lim _{x \downarrow 0}\left((A+x I)^{-1} f, f\right)=\lim _{x \downarrow 0}\left\|\left(A_{o}+x I\right)^{-\frac{1}{2}} P_{A} f\right\|^{2} \\
& =\left\{\begin{array}{ll}
0, & f \in \operatorname{mul} A \\
\left\|A_{o}^{\left[-\frac{1}{2}\right]} P_{A} f\right\|^{2}, & P_{A} f \in \operatorname{ran} A_{o}^{\frac{1}{2}} \\
+\infty, & f \in \mathcal{H} \backslash \operatorname{ran} A^{\frac{1}{2}}
\end{array}=\left\{\begin{array}{ll}
A^{-1}[f], & f \in \operatorname{ran} A^{\frac{1}{2}} \\
+\infty, & f \in \mathcal{H} \backslash \operatorname{ran} A^{\frac{1}{2}}
\end{array} .\right.\right.
\end{aligned}
$$


Let $A$ and $B$ be two nonnegative selfadjoint l.r.. If $\operatorname{dom} A \cap \operatorname{dom} B \neq 0$, then it is naturally defined the sum

$$
A+B:=\left\{\left\{f, f^{\prime}+g^{\prime}\right\}:\left\{f, f^{\prime}\right\} \in A,\left\{f, g^{\prime}\right\} \in B\right\}
$$

which is nonnegative symmetric l.r. and it, in general, is not selfadjoint.

On the other side, one can define a quadratic form

$$
\mathfrak{g}[\varphi]=A[\varphi]+B[\varphi], \varphi \in \mathcal{D}[A] \cap \mathcal{D}[B],
$$

which is nonnegative and closed [33, 25, 29]. Hence, by the first representation theorem, [33, 46] there is a nonnegative selfadjoint l.r. associated with $\mathfrak{g}$. This l.r. is called the form sum of $A$ and $B$ [24, 25, 29, 33] and is denoted by $A \dot{+} B$. Observe that

$$
A \dot{+} B=\{0\} \oplus \mathcal{H} \Longleftrightarrow \mathcal{D}[A] \cap \mathcal{D}[B]=\{0\} .
$$

\section{ShORTED OPERATORS FOR NON-NEGATIVE SELFADJOINT LINEAR RELATIONS}

Theorem 3.1. Let $A$ be a nonnegative selfadjoint l.r. in the Hilbert space $\mathcal{H}$ and let $\mathcal{L}$ be a subspace of $\mathcal{H}$. Then the set

$$
\Xi(A, \mathcal{L})=\{\widetilde{A} \text { is a nonnegative selfadjoint l.r., } \widetilde{A} \leq A, \operatorname{ran} \widetilde{A} \subseteq \mathcal{L}\}
$$

has a unique maximal element $A_{\mathcal{L}}$ and $\operatorname{ker} A_{\mathcal{L}} \supseteq \mathcal{L}^{\perp}$. Moreover,

(1) the l.r. $A_{\mathcal{L}}\left\lceil\mathcal{L}\right.$ is selfadjoint in $\mathcal{L}$ and $\left(A_{\mathcal{L}}\lceil\mathcal{L})^{-1}\right.$ is associated with the closed sesquilinear form $\mathfrak{c}_{\mathcal{L}}$ defined as follows

$$
\mathfrak{c}_{\mathcal{L}}[f, g]:=A^{-1}[f, g], f, g \in \operatorname{dom} \mathfrak{c}_{\mathcal{L}}=\operatorname{ran} A^{\frac{1}{2}} \cap \mathcal{L}
$$

(2) the equality $\operatorname{ran}\left(A_{\mathcal{L}}\right)^{\frac{1}{2}}=\operatorname{ran} A^{\frac{1}{2}} \cap \mathcal{L}$ holds and $A_{\mathcal{L}}=0$ (dom $A_{\mathcal{L}}=\mathcal{H}$ ) if and only if $\operatorname{ran} A^{\frac{1}{2}} \cap \mathcal{L}=\{0\}$

(3) if $\mathfrak{C}(A)$ is the Cayley transform (1.12) of $A$, then

$$
A_{\mathcal{L}}=\mathfrak{C}\left(I-(I-\mathfrak{C}(A))_{\mathcal{L}}\right)
$$

(4) $A_{\mathcal{L}}$ is an operator (mul $A_{\mathcal{L}}=\{0\}$ ) if and only if mul $A \cap \mathcal{L}=\{0\}$;

(5) $A_{\mathcal{L}} \in \mathbf{B}^{+}(\mathcal{H}) \backslash\{0\}$ if and only if the linear manifold

$$
\mathfrak{M}:=\left\{f \in \mathcal{D}[A]: A^{\frac{1}{2}} f \in \mathcal{L}\right\}
$$

is a subspace and mul $A \cap \mathcal{L}=\{0\}$.

Proof. The operator $T:=\mathfrak{C}(A)$ admits the following block operator matrix representation

$$
T=\left[\begin{array}{cc}
D & C \\
C^{*} & F
\end{array}\right]: \begin{array}{lll}
\mathcal{L}^{\perp} & & \mathcal{L}^{\perp} \\
& \mathcal{L}
\end{array} \rightarrow \begin{gathered}
\oplus \\
\mathcal{L}
\end{gathered}
$$

If $\widetilde{A} \in \Xi(A, \mathcal{L})$ and if $\widetilde{T}=\mathfrak{C}(\widetilde{A})=-I+2(I+\widetilde{A})^{-1}$ is the Cayley transform of $\widetilde{A}$, then because $\widetilde{A}+I \leq A+I$ and $2(\widetilde{A}+I)^{-1}=I+\widetilde{T}, 2(A+I)^{-1}=I+T$, we conclude that $I+\widetilde{T} \geq I+T$. On the other hand, the representation

$$
\widetilde{A}=\{\{(I+\widetilde{T}) g,(I-\widetilde{T}) g\}, g \in \mathcal{H}\}
$$


and the inclusion $\operatorname{ran} \widetilde{A} \subseteq \mathcal{L}$ yield that $\operatorname{ran}(I-\widetilde{T}) \subseteq \mathcal{L}$. The latter is equivalent to the equality $\widetilde{T}\left\lceil\mathcal{L}^{\perp}=I_{\mathcal{L}^{\perp}}\right.$. It follows that $\widetilde{T}$ is of the form $\widetilde{T}=\left[\begin{array}{ll}I & 0 \\ 0 & \widetilde{F}\end{array}\right]: \begin{array}{l}\mathcal{L}^{\perp} \\ \begin{array}{l}\oplus \\ \mathcal{L}\end{array}\end{array} \quad \begin{array}{l}\mathcal{L}^{\perp} \\ \begin{array}{l}\oplus \\ \mathcal{L}\end{array}\end{array}$. Since

$$
(I+\widetilde{T})-(I+T)=\left[\begin{array}{cc}
I-D & -C \\
-C^{*} & \widetilde{F}-F
\end{array}\right]: \begin{array}{ccc}
\mathcal{L}^{\perp} & \mathcal{L}^{\perp} \\
\mathcal{L} & \rightarrow & \oplus \\
\mathcal{L}
\end{array}
$$

and $(I+\widetilde{T})-(I+T) \geq 0$, from (1.4) we get the following inequality

$$
\widetilde{F}-F-\left((I-D)^{\left[-\frac{1}{2}\right]} C\right)^{*}(I-D)^{\left[-\frac{1}{2}\right]} C \geq 0 .
$$

Recall (cf. [15, 20, 48]), that the block operator-matrix (3.5) is a selfadjoint contraction if and only if the following properties of the entries are valid

$$
\left\{\begin{array}{l}
D \in\left[-I_{\mathcal{L}^{\perp}}, I_{\mathcal{L}^{\perp}}\right], C^{*}=N D_{D}, F=-N D N^{*}+D_{N^{*}} X D_{N^{*}}, \\
N \in \mathbf{B}\left(\mathfrak{D}_{D}, \mathcal{L}\right) \text { is a contraction, } \\
X \in \mathbf{B}\left(\mathfrak{D}_{N^{*}}\right) \text { is a selfadjoint contraction }
\end{array} .\right.
$$

From the structure (3.6) of entries of $T$ we obtain that

$$
F^{\prime}:=F+\left((I-D)^{\left[-\frac{1}{2}\right]} C\right)^{*}(I-D)^{\left[-\frac{1}{2}\right]} C=N N^{*}+D_{N^{*}} X D_{N^{*}} .
$$

Thus, $-I_{\mathcal{L}} \leq F^{\prime} \leq \widetilde{F} \leq I_{\mathcal{L}}$. Since the set $\Xi(A, \mathcal{L})$ coincides with the Cayley transforms of the set

$$
\Upsilon(T, \mathcal{L}):=\mathfrak{C}(\Xi(A, \mathcal{L}))=\left\{\widetilde{T} \in[-I, I]: \widetilde{T} \uparrow \mathcal{L}^{\perp}=I_{\mathcal{L}^{\perp}}, \widetilde{T} \geq T\right\}
$$

and

$$
\min _{\widetilde{T} \in \Upsilon(B \mathcal{L})} \widetilde{T}=T^{\prime}=\left[\begin{array}{cc}
I & 0 \\
0 & F^{\prime}
\end{array}\right]: \begin{gathered}
\mathcal{L}^{\perp} \\
\underset{\mathcal{L}}{\oplus}
\end{gathered} \quad \begin{gathered}
\mathcal{L}^{\perp} \\
\mathcal{L}
\end{gathered}
$$

we obtain that

$$
\max _{\widetilde{A} \in \Xi(A, \mathcal{L})} \widetilde{A}=\mathfrak{C}\left(T^{\prime}\right)=: A_{\mathcal{L}}
$$

Let $(I-T)_{\mathcal{L}}$ be the Kreŭn shorted operator. Then (3.5) and (1.5) yield

$$
(I-T)_{\mathcal{L}}=\left[\begin{array}{cc}
0 & 0 \\
0 & I-F-\left((I-D)^{\left[-\frac{1}{2}\right]} C\right)^{*}(I-D)^{\left[-\frac{1}{2}\right]} C
\end{array}\right]=\left[\begin{array}{cc}
0 & 0 \\
0 & I-F^{\prime}
\end{array}\right] .
$$

Therefore

$$
T^{\prime}=I-(I-T)_{\mathcal{L}}, A_{\mathcal{L}}=\mathfrak{C}\left(I-(I-T)_{\mathcal{L}}\right) .
$$

Due to the properties of the shorted operator we have

$$
\mathcal{L} \cap \operatorname{ran}(I-T)^{\frac{1}{2}}=\operatorname{ran}\left((I-T)_{\mathcal{L}}\right)^{\frac{1}{2}}=\operatorname{ran}\left(I-F^{\prime}\right)^{\frac{1}{2}}
$$

It follows that

$$
\begin{aligned}
A_{\mathcal{L}} & =\mathfrak{C}\left(T^{\prime}\right)=\left\{\left\{\left(I+T^{\prime}\right) g,\left(I-T^{\prime}\right) g\right\}, g \in \mathcal{H}\right\} \\
& =\left\{\{h, 0\}: h \in \mathcal{L}^{\perp}\right\} \bigoplus\left\{\left\{\left(I_{\mathcal{L}}+F^{\prime}\right) f,\left(I_{\mathcal{L}}-F^{\prime}\right) f\right\}: f \in \mathcal{L}\right\}=\left(\mathcal{L}^{\perp} \oplus\{0\}\right) \bigoplus \mathfrak{C}\left(F^{\prime}\right) .
\end{aligned}
$$


Hence $A_{\mathcal{L}}\left\lceil\mathcal{L}=\mathfrak{C}\left(F^{\prime}\right) \subset \mathcal{L} \oplus \mathcal{L}\right.$. Set

$$
\mathcal{L}^{\prime}:=\left\{f \in \mathcal{H}:(I-T)^{\frac{1}{2}} f \in \mathcal{L}\right\} .
$$

Because (see (1.2)),

$$
(I-T)_{\mathcal{L}}=(I-T)^{\frac{1}{2}} P_{\mathcal{L}^{\prime}}(I-T)^{\frac{1}{2}}
$$

and

we get that

$$
(I-T)_{\mathcal{L}} \mid \mathcal{L}=I-F^{\prime}
$$

$$
(I-T)^{\frac{1}{2}} \uparrow \mathcal{L}^{\prime}=\left(I-F^{\prime}\right)^{\frac{1}{2}} V^{\prime},
$$

where $V^{\prime}$ is an isometry from $\mathcal{L}^{\prime}$ onto $\overline{\operatorname{ran}}\left(I-F^{\prime}\right)$. It follows that

$$
\left\|(I-T)^{\left[-\frac{1}{2}\right]} f\right\|=\left\|\left(I-F^{\prime}\right)^{\left[-\frac{1}{2}\right]} f\right\|, f \in \operatorname{ran}(I-T)^{\frac{1}{2}} \cap \mathcal{L}=\operatorname{ran}\left(I-F^{\prime}\right)^{\frac{1}{2}} .
$$

Now (2.7) yields that

$$
A^{-1}[f, g]=\left(\mathfrak{C}\left(F^{\prime}\right)\right)^{-1}[f, g]=\left(A_{\mathcal{L}}\lceil\mathcal{L})^{-1}[f, g], f, g \in \mathcal{D}\left[A^{-1}\right] \cap \mathcal{L}=\operatorname{ran} A^{\frac{1}{2}} \cap \mathcal{L} .\right.
$$

Using definition $(3.2)$ of the form $\mathfrak{c}_{\mathcal{L}}$, we get

$$
\begin{gathered}
\operatorname{mul} A_{\mathcal{L}}=\{0\} \Longleftrightarrow \operatorname{ker}\left(A_{\mathcal{L}}\right)^{-1}=\{0\} \Longleftrightarrow \operatorname{ker} \mathfrak{c}_{\mathcal{L}}=\{0\} \Longleftrightarrow \operatorname{mul} A \cap \mathcal{L}=\{0\}, \\
A_{\mathcal{L}} \in \mathbf{B}^{+}(\mathcal{H}) \backslash\{0\} \Longleftrightarrow\left\{\begin{array}{l}
\operatorname{mul} A \cap \mathcal{L}=\{0\}, \\
\mathfrak{c}_{\mathcal{L}}[f] \geq m\|f\|^{2} \forall f \in \mathcal{L} \cap \operatorname{ran} A_{o}^{\frac{1}{2}}, m>0
\end{array}\right.
\end{gathered}
$$

The latter is equivalent to the conditions: $\operatorname{mul} A \cap \mathcal{L}=\{0\}$ and the linear manifold defined in (3.4) is closed.

Observe that since $A_{\mathcal{L}} \leq A$ and $\left.\mathcal{D}\left[A_{\mathcal{L}}\right]=\mathcal{L}^{\perp} \oplus D\left[A_{\mathcal{L}}\right\rceil \mathcal{L}\right]$, we have

$$
D[A] \cap \mathcal{L} \subseteq D\left[A_{\mathcal{L}}\lceil\mathcal{L}], A_{\mathcal{L}}[f] \leq A[f] \quad \forall f \in D[A] \cap \mathcal{L}\right.
$$

Let $A_{1}$ and $A_{2}$ be nonnegative selfadjoint l.r.. Suppose $A_{1} \leq A_{2}$. Then $\Xi\left(A_{1}, \mathcal{L}\right) \subset \Xi\left(A_{2}, \mathcal{L}\right)$ for any subspace $\mathcal{L}$ in $\mathfrak{H}$. Hence

$$
\left(A_{1}\right)_{\mathcal{L}} \leq\left(A_{2}\right)_{\mathcal{L}}
$$

The next proposition is an extension of the property (1.6) to the set of all nonnegative selfadjoint l.r..

Proposition 3.2. Let $\left\{A_{n}\right\}$ be a sequences of nonnegative selfadjoint l.r. and let $\mathcal{L}$ be a subspace in $\mathcal{H}$. Then in the strong resolvent convergence sense

$$
A_{n} \searrow A_{\infty} \Longrightarrow\left(A_{n}\right)_{\mathcal{L}} \searrow\left(A_{\infty}\right)_{\mathcal{L}}
$$

Proof. Set $T_{n}:=\mathfrak{C}\left(A_{n}\right), n \in \mathbb{N}, T_{\infty}:=\mathfrak{C}\left(A_{\infty}\right)$. Then

$$
\mathrm{s}-\mathrm{R}-\lim _{n \rightarrow \infty} A_{n}=A_{\infty} \Longrightarrow \mathrm{s}-\lim _{n \rightarrow \infty} T_{n}=T_{\infty}
$$

Moreover, $\left\{A_{n}\right\}$ is non-increasing $\Longleftrightarrow\left\{T_{n}\right\}$ is non-decreasing $\Longleftrightarrow\left\{I-T_{n}\right\}$ is non-increasing $\Longrightarrow\left\{\left(I-T_{n}\right)_{\mathcal{L}}\right\}$ is non-increasing for each subspace $\mathcal{L}$ in $\mathcal{H}$. Because $\left\{I-T_{n}\right\} \subset \mathbf{B}^{+}(\mathcal{H})$ we get [47] that in the strong convergence sense

$$
\left(I-T_{n}\right)_{\mathcal{L}} \searrow\left(I-T_{\infty}\right)_{\mathcal{L}}
$$

Hence from (3.3)

$$
\mathrm{s}-\mathrm{R}-\lim _{n \rightarrow \infty}\left(A_{n}\right)_{\mathcal{L}}=\mathrm{s}-\mathrm{R}-\lim _{n \rightarrow \infty} \mathfrak{C}\left(I-\left(I-T_{n}\right)_{\mathcal{L}}\right)=\mathfrak{C}\left(I-\left(I-T_{\infty}\right)_{\mathcal{L}}\right)=\left(A_{\infty}\right)_{\mathcal{L}}
$$

The proof is complete. 
Replacing $A$ by $A^{-1}$ in Theorem 3.1 we get that

$$
\left(A^{-1}\right)_{\mathcal{L}}=\max \left\{\widetilde{B}: \widetilde{B} \in \Xi\left(A^{-1}, \mathcal{L}\right)\right\}=\min \left\{\widetilde{A}: \widetilde{A}=\widetilde{A}^{*}, \widetilde{A} \geq A, \operatorname{dom} \widetilde{A} \subseteq \mathcal{L}\right\}
$$

and $\left(\left(A^{-1}\right)_{\mathcal{L}}\lceil\mathcal{L})^{-1}\right.$ is associated with the closed form

$$
\mathfrak{d}_{\mathcal{L}}[\varphi, \psi]=A[\varphi, \psi], \varphi, \psi \in \mathcal{D}[A] \cap \mathcal{L}
$$

Besides, $\operatorname{ran}\left(\left(A^{-1}\right)_{\mathcal{L}}\right)^{\frac{1}{2}}=\mathcal{D}[A] \cap \mathcal{L}$ and $\left(A^{-1}\right)_{\mathcal{L}}=0$ if and only if $\mathcal{D}[A] \cap \mathcal{L}=\{0\}$.

Theorem 3.3. Let $A$ be a nonnegative selfadjoint l.r. in the Hilbert space $\mathcal{H}$ and let $\mathcal{L}$ be a subspace of $\mathcal{H}$. Then

$$
A_{\mathcal{L}} \leq\left(\left(A^{-1}\right)_{\mathcal{L}}\right)^{-1}
$$

Let $\mathcal{M}_{A, \mathcal{L}}(\lambda)$ be the Nevanlinna family defined in (2.1). Then

$$
\begin{aligned}
& A_{\mathcal{L}} \uparrow \mathcal{L}=-\left(\mathrm{s}-\mathrm{R}-\lim _{\lambda \uparrow 0} \mathcal{M}_{A, \mathcal{L}}(\lambda)\right), \\
& \left(\left(A^{-1}\right)_{\mathcal{L}}\right)^{-1} \uparrow \mathcal{L}=-\left(\mathrm{s}-\mathrm{R}-\lim _{\lambda \downarrow-\infty} \mathcal{M}_{A, \mathcal{L}}(\lambda)\right) .
\end{aligned}
$$

Proof. If $\mathfrak{C}(A)=T$, then $\mathfrak{C}\left(A^{-1}\right)=-T$. Hence from (3.3) we get

$$
\left(A^{-1}\right)_{\mathcal{L}}=\mathfrak{C}\left(I-(I+T)_{\mathcal{L}}\right) .
$$

Then (3.8) implies

$$
\left(\left(A^{-1}\right)_{\mathcal{L}}\right)^{-1}=\mathfrak{C}\left((I+T)_{\mathcal{L}}-I\right) .
$$

The equality $(I-T)+(I+T)=2 I$ and the definition of the shorted operator produce the inequality

Hence

$$
(I-T)_{\mathcal{L}}+(I+T)_{\mathcal{L}} \leq 2 I
$$

From (2.8) it follows that

$$
I-(I-T)_{\mathcal{L}} \geq(I+T)_{\mathcal{L}}-I
$$

$$
A_{\mathcal{L}}=\mathfrak{C}\left(I-(I-T)_{\mathcal{L}}\right) \leq \mathfrak{C}\left((I+T)_{\mathcal{L}}-I\right)=\left(\left(A^{-1}\right)_{\mathcal{L}}\right)^{-1}
$$

The operator $T$ takes the block operator matrix form (3.5). The entries $C$ and $F$ of $T$ admit the representations (3.6), i.e.,

$$
C^{*}=N D_{D}, C=D_{D} N^{*}, F=-N D N^{*}+D_{N^{*}} X D_{N^{*}} .
$$

Define the function

$$
\Phi(z)=F+z C^{*}\left(I_{\mathcal{L}^{\perp}}-z D\right)^{-1} C, z \in \mathbb{C} \backslash\{(-\infty,-1] \cup[1,+\infty)\} .
$$

The function $\Phi$ belongs to the class $\mathcal{R} \mathcal{S}(\mathcal{L})[13$, i.e., $\Phi$ is a Nevanlinna function and the Schur class function in the unit disk $\mathbb{D}$. Then there exist strong limit values $\Phi( \pm 1)$ and

$$
\begin{aligned}
& \Phi(-1)=s-\lim _{x \downarrow-1} \Phi(x)=F-\left((I+D)^{\left[-\frac{1}{2}\right]} C\right)^{*}(I+D)^{\left[-\frac{1}{2}\right]} C=-N N^{*}+D_{N^{*}} X D_{N^{*}}, \\
& \Phi(1)=s-\lim _{x \uparrow 1} \Phi(x)=F+\left((I-D)^{\left[-\frac{1}{2}\right]} C\right)^{*}(I-D)^{\left[-\frac{1}{2}\right]} C=N N^{*}+D_{N^{*}} X D_{N^{*}} .
\end{aligned}
$$

Observe that

$$
(I \pm T)_{\mathcal{L}}=\left[\begin{array}{cc}
0 & 0 \\
0 & I \pm \Phi(\mp 1)
\end{array}\right]: \begin{gathered}
\mathcal{L}^{\perp} \\
\underset{\mathcal{L}}{\oplus} \rightarrow \frac{\mathcal{L}^{\perp}}{\oplus}
\end{gathered}
$$


Therefore (3.3) and (3.9) yield

$$
A_{\mathcal{L}} \uparrow \mathcal{L}=\mathfrak{C}(\Phi(1)),\left(\left(A^{-1}\right)_{\mathcal{L}}\right)^{-1} \uparrow \mathcal{L}=\mathfrak{C}(\Phi(-1)) .
$$

But then due to $\left.\Phi(1)=\mathfrak{C}\left(A_{\mathcal{L}}\right\rceil \mathcal{L}\right)$ and $\Phi(-1)=\mathfrak{C}\left(\left(\left(A^{-1}\right)_{\mathcal{L}}\right)^{-1} \uparrow \mathcal{L}\right)$ we have $-\Phi(1)=$ $\mathfrak{C}\left(\left(A_{\mathcal{L}}\lceil\mathcal{L})^{-1}\right)\right.$

$$
I_{\mathcal{L}}-\Phi(1)=2\left(I_{\mathcal{L}}+\left(A_{\mathcal{L}} \uparrow \mathcal{L}\right)^{-1}\lceil\mathcal{L})^{-1}, I_{\mathcal{L}}+\Phi(-1)=2\left(I_{\mathcal{L}}+\left(\left(A^{-1}\right)_{\mathcal{L}}\right)^{-1} \uparrow \mathcal{L}\right)^{-1}\right.
$$

Note that according to the Schur-Frobenius formula for the resolvent of block-operator matrix the relation

$$
P_{\mathcal{L}}\left(I_{\mathcal{H}}-z T\right)^{-1}\left\lceil\mathcal{L}=\left(I_{\mathcal{L}}-z \Phi(z)\right)^{-1}, z \in \mathbb{C} \backslash\{(-\infty,-1] \cup[1,+\infty)\}\right.
$$

holds.

Further we will use the following notations

$$
\begin{aligned}
& \mathcal{N}_{A, \mathcal{L}}(\lambda):=P_{\mathcal{L}}(A-\lambda I)^{-1} \uparrow \mathcal{L}, \lambda \in \mathbb{C} \backslash \mathbb{R}_{+}, \\
& \mathcal{N}_{T, \mathcal{L}}(\xi):=P_{\mathcal{L}}(T-\xi I)^{-1} \uparrow \mathcal{L}, \xi \in \mathbb{C} \backslash[-1,1] .
\end{aligned}
$$

The resolvents of $A$ and $T$ are connected by the relations

$$
\begin{aligned}
& \left\{\begin{array}{l}
(A-\lambda I)^{-1}=-\frac{1}{1+\lambda}\left(I+\frac{2}{1+\lambda}\left(T-\frac{1-\lambda}{1+\lambda} I\right)^{-1}\right), \lambda \in \rho(A) \backslash\{-1\} \\
(A+I)^{-1}=\frac{1}{2}(T+I),
\end{array},\right. \\
& \Longleftrightarrow(A-\lambda I)^{-1}=\frac{1}{1-\lambda}(T+I)\left(I-\frac{1+\lambda}{1-\lambda} T\right)^{-1}, \lambda \in \rho(A) .
\end{aligned}
$$

Hence

$$
\begin{aligned}
&\left(\mathcal{N}_{A, \mathcal{L}}^{-1}(\lambda)+I\right)^{-1}=\mathcal{N}_{A, \mathcal{L}}(\lambda)\left(\mathcal{N}_{A, \mathcal{L}}(\lambda)+I\right)^{-1} \\
&=-\left(I+\frac{2}{1+\lambda} \mathcal{N}_{T, \mathcal{L}}\left(\frac{1-\lambda}{1+\lambda}\right)\right)\left(\lambda-\frac{2}{1+\lambda} \mathcal{N}_{T, \mathcal{L}}\left(\frac{1-\lambda}{1+\lambda}\right)\right)^{-1} \\
& \quad=-\left(\mathcal{N}_{T, \mathcal{L}}^{-1}\left(\frac{1-\lambda}{1+\lambda}\right)+\frac{2}{1+\lambda}\right)\left(\lambda \mathcal{N}_{T, \mathcal{L}}^{-1}\left(\frac{1-\lambda}{1+\lambda}\right)-\frac{2}{1+\lambda}\right)^{-1} .
\end{aligned}
$$

From the equality $\mathfrak{C}\left(A^{-1}\right)=-T$ and the equality

$$
\mathcal{N}_{-T, \mathcal{L}}^{-1}\left(\frac{1-\lambda}{1+\lambda}\right)=-\mathcal{N}_{T, \mathcal{L}}^{-1}\left(-\frac{1-\lambda}{1+\lambda}\right)
$$

one gets

$$
\begin{aligned}
\left(\mathcal{N}_{A^{-1}, \mathcal{L}}^{-1}(\lambda)\right. & +I)^{-1} \\
& =-\left(-\mathcal{N}_{T, \mathcal{L}}^{-1}\left(-\frac{1-\lambda}{1+\lambda}\right)+\frac{2}{1+\lambda}\right)\left(-\lambda \mathcal{N}_{T, \mathcal{L}}^{-1}\left(-\frac{1-\lambda}{1+\lambda}\right)-\frac{2}{1+\lambda}\right)^{-1} .
\end{aligned}
$$

Using (3.11) in the form

$$
\mathcal{N}_{T, \mathcal{L}}(\xi)=\left(\Phi\left(\xi^{-1}\right)-\xi I\right)^{-1}, \xi \in \mathbb{C} \backslash[-1,1],
$$

where $\Phi(\cdot)$ is defined by (3.10), we get the equalities

$$
s-\lim _{\xi \downarrow 1} \mathcal{N}_{T, \mathcal{L}}^{-1}(\xi)=\Phi(1)-I_{\mathcal{L}}, s-\lim _{\xi \uparrow-1} \mathcal{N}_{T, \mathcal{L}}^{-1}(\xi)=I_{\mathcal{L}}+\Phi(-1) .
$$


Using (3.12), (3.13) and since

$$
2\left(I+\left(A_{\mathcal{L}}\right)^{-1} \uparrow \mathcal{L}\right)^{-1}=I-\Phi(1), 2\left(I+\left(\left(A^{-1}\right)_{\mathcal{L}}\right)^{-1} \uparrow \mathcal{L}\right)^{-1}=I+\Phi(-1),
$$

we have

$$
\begin{aligned}
& A_{\mathcal{L}}\left\lceil\mathcal{L}=\mathrm{s}-\mathrm{R}-\lim _{\lambda \uparrow 0}\left(P_{\mathcal{L}}(A-\lambda)^{-1} \uparrow \mathcal{L}\right)^{-1}\right. \\
& \left(A^{-1}\right)_{\mathcal{L}}=\mathrm{s}-\mathrm{R}-\lim _{\lambda \uparrow 0}\left(P_{\mathcal{L}}\left(A^{-1}-\lambda\right)^{-1}\lceil\mathcal{L})^{-1}\right.
\end{aligned}
$$

From (2.1)

$$
\left(P_{\mathcal{L}}(A-\lambda)^{-1}\lceil\mathcal{L})^{-1}=-\mathcal{M}_{A, \mathcal{L}}(\lambda)-\lambda I_{\mathcal{L}}, \lambda \in \rho(A) .\right.
$$

Hence

Similarly

$$
A_{\mathcal{L}}\left\lceil\mathcal{L}=-\left(\mathrm{s}-\mathrm{R}-\lim _{\lambda \uparrow 0} \mathcal{M}_{A, \mathcal{L}}(\lambda)\right)\right.
$$

Consequently

$$
\left(A^{-1}\right)_{\mathcal{L}} \uparrow \mathcal{L}=-\left(\mathrm{s}-\mathrm{R}-\lim _{\lambda \uparrow 0} \mathcal{M}_{A^{-1}, \mathcal{L}}(\lambda)\right)
$$

Now (2.2) yields that

$$
\left(\left(\left(A^{-1}\right)_{\mathcal{L}}\right)\lceil\mathcal{L})^{-1}=-\left(\mathrm{s}-\mathrm{R}-\lim _{\lambda \uparrow 0}\left(\mathcal{M}_{A^{-1}, \mathcal{L}}(\lambda)\right)^{-1}\right)\right.
$$

$$
\begin{aligned}
\left(\left(\left(A^{-1}\right)_{\mathcal{L}} \backslash \mathcal{L}\right)^{-1}=-\left(\mathrm{s}-\mathrm{R}-\lim _{\mu \downarrow-\infty} \mathcal{M}_{A, \mathcal{L}}(\mu)\right)\right. & \\
\quad & =\mathrm{s}-\mathrm{R}-\lim _{\mu \downarrow-\infty}\left(\mu I_{\mathcal{L}}+\left(P_{\mathcal{L}}(A-\mu I)^{-1}\lceil\mathcal{L})^{-1}\right) .\right.
\end{aligned}
$$

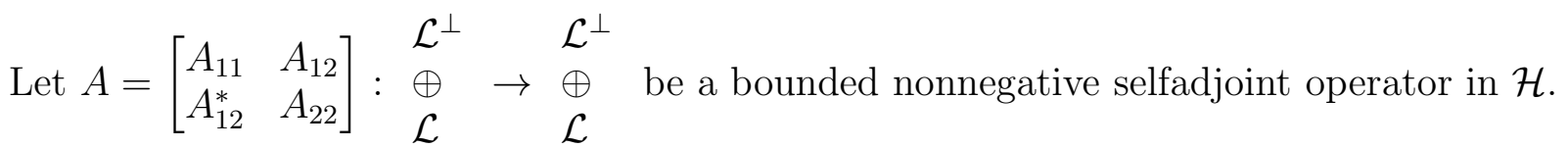

Due to (1.4) the entry $A_{12}$ admits the representation

$$
A_{12}=A_{11}^{\frac{1}{2}} Y A_{22}^{\frac{1}{2}}, Y \in \mathbf{B}\left(\overline{\operatorname{ran}} A_{22}, \overline{\operatorname{ran}} A_{11}\right) \quad \text { is a contraction. }
$$

From (1.5) we get the equality $A_{\mathcal{L}}=A_{22}^{\frac{1}{2}}\left(I-Y^{*} Y\right) A_{22}^{\frac{1}{2}}$. In general $A^{-1}$ is a l.r.. But $\left(\left(A^{-1}\right)_{\mathcal{L}}\right)^{-1}$ is associated with the form $A[\varphi, \psi], \varphi, \psi \in \mathcal{L}$. Hence

$$
\left(\left(A^{-1}\right)_{\mathcal{L}}\lceil\mathcal{L})^{-1}=A_{22}\right.
$$

So, if $A_{22}$ has bounded inverse, then $\left(A^{-1}\right)_{\mathcal{L}}$ is bounded and vice versa. If this is a case, then $\left(A^{-1}\right)_{\mathcal{L}}=A_{22}^{-1}$.

On the other side the function $\mathcal{M}_{A, \mathcal{L}}$ defined in (2.3) takes the form

$$
\mathcal{M}_{A, \mathcal{L}}(\lambda)=-A_{22}+A_{22}^{\frac{1}{2}} Y^{*}\left(A_{11}-\lambda I_{\mathcal{L}^{\perp}}\right)^{-1} A_{11} Y A_{22}^{\frac{1}{2}}, \lambda \in \rho\left(A_{11}\right) .
$$

Hence

$$
\begin{aligned}
& -\left(\mathrm{s}-\lim _{\lambda \uparrow 0} \mathcal{M}(\lambda)\right)=A_{22}^{\frac{1}{2}}\left(I-Y^{*} Y\right) A_{22}^{\frac{1}{2}}=A_{\mathcal{L}}\lceil\mathcal{L}, \\
& -\left(\mathrm{s}-\lim _{\lambda \downarrow-\infty} \mathcal{M}(\lambda)\right)=A_{22}=\left(\left(A^{-1}\right)_{\mathcal{L}}\lceil\mathcal{L})^{-1}\right.
\end{aligned}
$$


Remark 3.4. Suppose that $\operatorname{codim} \mathcal{L}<\infty$. Let $A$ be a nonnegative selfadjoint l.r. in $\mathcal{H}$. Then due to [50, 16] the l.r. $P_{\mathcal{L}} A^{-1} \uparrow \mathcal{L}$ and $P_{\mathcal{L}} A\lceil\mathcal{L}$ are selfadjoint and, therefore, they are associated with the closed forms $\mathfrak{c}_{\mathcal{L}}$ and $\mathfrak{d}_{\mathcal{L}}$ defined in (3.2) and in (3.7), respectively. Hence, due to Theorem 3.1 one gets

$$
A_{\mathcal{L}}\left\lceil\mathcal{L}=\left(P_{\mathcal{L}} A^{-1}\lceil\mathcal{L})^{-1},\left(\left(A^{-1}\right)_{\mathcal{L}}\right)^{-1}=P_{\mathcal{L}} A\lceil\mathcal{L}\right.\right.
$$

\section{Parallel addition of NOnNEGative SELFAdjoint Linear RELATiOns}

Definition 4.1. Let $A$ and $B$ be nonnegative selfadjoint l.r. in $\mathcal{H}$. Then the nonnegative selfadjoint l.r. $A: B$ defined as follows

$$
A: B=\left(A^{-1} \dot{+} B^{-1}\right)^{-1},
$$

where $\dot{+}$ is the form sum, is said to be the parallel sum of $A$ and B. The l.r.

$$
h(A, B):=2(A: B)=\left(\frac{A^{-1} \dot{+} B^{-1}}{2}\right)^{-1}
$$

is said to be the harmonic mean of $A$ and $B$.

Clearly, the parallel sum of two nonnegative selfadjoint l.r. is the nonnegative selfadjoint l.r. as well. From Definition 4.1 it follows that

$$
\begin{aligned}
& A^{-1} \dot{+} B^{-1}=(A: B)^{-1}, A^{-1}: B^{-1}=(A \dot{+} B)^{-1}, \\
& \frac{1}{2}\left(A^{-1} \dot{+} B^{-1}\right)=(h(A, B))^{-1}, h\left(A^{-1}, B^{-1}\right)=\left(\frac{A \dot{+} B}{2}\right)^{-1},
\end{aligned}
$$

and

$$
\operatorname{mul}(A: B)=\operatorname{ker}\left(A^{-1} \dot{+} B^{-1}\right)=\operatorname{mul} A \cap \operatorname{mul} B .
$$

Hence, $A: B$ is a selfadjoint operator if and only if mul $A \cap \operatorname{mul} B=\{0\}$.

The parallel sum $A: B$ is a bounded operator if and only if mul $A \cap \operatorname{mul} B=\{0\}$ and the quadratic form

$$
\mathfrak{q}[g]:=A^{-1}[g]+B^{-1}[g], g \in \operatorname{ran} A^{\frac{1}{2}} \cap \operatorname{ran} B^{\frac{1}{2}}
$$

is positive definite. In particular, if $A$ or $B$ is a bounded nonnegative selfadjoint operator, then the nonnegative selfadjoint l.r. $A^{-1} \dot{+} B^{-1}$ has a bounded inverse, hence in this case the l.r. $A: B$ is a bounded nonnegative selfadjoint operator.

The theorem below shows that such way defined parallel addition for nonnegative selfadjoint l.r. preserves basic properties of parallel addition for bounded nonnegative selfadjoint linear operators.

Theorem 4.2. The parallel sum of nonnegative selfadjoint l.r. possesses the properties:

(1) $\lambda A: \lambda B=\lambda(A: B), \lambda>0$,

(2) $A: B=B: A$

(3) $(A: B): C=A:(B: C)=\left(A^{-1} \dot{+} B^{-1} \dot{+} C^{-1}\right)^{-1}$,

(4) $A: B \leq A, A: B \leq B$,

(5) $A_{1} \leq \bar{A}_{2} \Longrightarrow A_{1}: \bar{B} \leq A_{2}: B$,

(6) $\operatorname{ran}(A: B)^{\frac{1}{2}}=\operatorname{ran} A^{\frac{1}{2}} \cap \operatorname{ran} B^{\frac{1}{2}}$,

(7) $A: B=0$ (i.e., $\operatorname{dom}(A: B)=\mathcal{H}$ and $(A: B) f=0 \forall f \in \mathcal{H}$ ) if and only if $\operatorname{ran} A^{\frac{1}{2}} \cap \operatorname{ran} B^{\frac{1}{2}}=\{0\}$

(8) if the quadratic form $A^{-1}[\cdot]$ is the closed restriction of the quadratic form $B^{-1}[\cdot]$, then

$$
h(A: B)=A \text {. }
$$


Besides, the following relation

$$
\begin{aligned}
A: B=\mathrm{s}-\mathrm{R}-\lim _{n \rightarrow \infty}\left(\left(A+\frac{1}{n} I\right):\left(B+\frac{1}{n} I\right)\right) & \\
& =\mathrm{s}-\mathrm{R}-\lim _{n \rightarrow \infty}\left(\left(A+\frac{1}{n} I\right)^{-1}+\left(B+\frac{1}{n} I\right)^{-1}\right)^{-1}
\end{aligned}
$$

is valid.

Proof. The first three properties follows directly from Definition 4.1. Because $A^{-1} \dot{+} B^{-1} \geq$ $A^{-1}$, we get $A: B=\left(A^{-1} \dot{+} B^{-1}\right)^{-1} \leq A$. Similarly, $A: B \leq B$.

$$
A: B=0 \Longleftrightarrow \operatorname{mul}\left(A^{-1} \dot{+} B^{-1}\right)=\mathcal{H} \Longleftrightarrow \operatorname{ran} A^{\frac{1}{2}} \cap \operatorname{ran} B^{\frac{1}{2}}=\{0\} .
$$

If $A_{1} \leq A_{2}$, then $A_{1}^{-1} \geq A_{2}^{-1}$ and $A_{1}^{-1} \dot{+} B^{-1} \geq A_{2}^{-1} \dot{+} B^{-1}$. Hence

$$
A_{1}: B=\left(A_{1}^{-1} \dot{+} B^{-1}\right)^{-1} \leq\left(A_{2}^{-1} \dot{+} B^{-1}\right)^{-1}=A_{2}: B \text {. }
$$

Further,

$$
\operatorname{ran}(A: B)^{\frac{1}{2}}=\operatorname{dom}\left(A^{-1} \dot{+} B^{-1}\right)^{\frac{1}{2}}=\operatorname{dom} A^{-\frac{1}{2}} \cap \operatorname{dom} B^{-\frac{1}{2}}=\operatorname{ran} A^{\frac{1}{2}} \cap \operatorname{ran} B^{\frac{1}{2}} .
$$

If the quadratic form $A^{-1}[\cdot]$ is the closed restriction of the quadratic form $B^{-1}[\cdot]$, then for the form sum $A^{-1} \dot{+} B^{-1}$ one has that $A^{-1} \dot{+} B^{-1}=2 A^{-1}$. Hence $A: B=\frac{1}{2} A$ and $h(A, B)=A$.

For each $n \in \mathbb{N}$ define the nonnegative selfadjoint l.r. $H_{n}$ as follows

$$
H_{n}=\left(\left(A+\frac{1}{n} I\right)^{-1}+\left(B+\frac{1}{n} I\right)^{-1}\right)^{-1}
$$

Then $\left\{H_{n}\right\}$ is a non-increasing sequence. By [17, Theorem 3.7] there exists

$$
H_{\infty}:=\mathrm{s}-\mathrm{R}-\lim _{n \rightarrow \infty} H_{n}=\mathrm{s}-\mathrm{R}-\lim _{n \rightarrow \infty}\left(\left(A+\frac{1}{n} I\right)^{-1}+\left(B+\frac{1}{n} I\right)^{-1}\right)^{-1}
$$

and

Since $H_{\infty} \leq H_{n} \forall n$, we get

$$
\begin{aligned}
& \operatorname{ran} H_{\infty}^{\frac{1}{2}}=\left\{g \in \bigcap_{n=1}^{\infty} \operatorname{ran} H_{n}^{\frac{1}{2}}: \lim _{n \rightarrow \infty}\left\|\left(H_{n}^{-\frac{1}{2}}\right)_{o} g\right\|^{2}<\infty\right\}, \\
& \left\|\left(H_{\infty}^{-\frac{1}{2}}\right)_{o} g\right\|^{2}=\lim _{n \rightarrow \infty}\left\|\left(H_{n}^{-\frac{1}{2}}\right)_{o} g\right\|^{2} .
\end{aligned}
$$

$$
H_{\infty} \leq A+\frac{1}{n} I, H_{\infty} \leq B+\frac{1}{n} I \quad \forall n \in \mathbb{N} .
$$

Hence $H_{\infty} \leq A$ and $H_{\infty} \leq B$.

Note that

$$
H_{n}^{-1}=\left(A+\frac{1}{n} I\right)^{-1}+\left(B+\frac{1}{n} I\right)^{-1}
$$

is a bounded nonnegative seldfadjoint operator in $\mathcal{H}$ for each $n \in \mathbb{N}$ and

$$
\left\|H_{n}^{-\frac{1}{2}} g\right\|^{2}=\left(\left(A+\frac{1}{n} I\right)^{-1} g, g\right)+\left(\left(B+\frac{1}{n} I\right)^{-1} g, g\right), g \in \mathcal{H} .
$$


Besides

$$
\operatorname{ker} H_{n}^{-1}=\operatorname{mul} A \cap \operatorname{mul} B \forall n \in \mathbb{N} \text {. }
$$

For each vector $h \in \mathcal{H}$ the sequence of numbers $\left\{\left\|H_{n}^{-\frac{1}{2}} g\right\|^{2}\right\}_{n=1}^{\infty}$ is non-decreasing. From (2.9) it follows that

$$
\lim _{n \rightarrow \infty}\left\|H_{n}^{-\frac{1}{2}} g\right\|^{2}<\infty \Longleftrightarrow g \in \operatorname{ran} A^{\frac{1}{2}} \cap \operatorname{ran} B^{\frac{1}{2}}
$$

Thus

$$
\begin{aligned}
& \operatorname{ran} H_{\infty}^{\frac{1}{2}}=\operatorname{ran} A^{\frac{1}{2}} \cap \operatorname{ran} B^{\frac{1}{2}}=\mathcal{D}\left[A^{-1}\right] \cap \mathcal{D}\left[B^{-1}\right] \\
& \left\|H_{\infty}^{-\frac{1}{2}} g\right\|^{2}=H_{\infty}^{-1}[g]=A^{-1}[g]+B^{-1}[g]=\left(A^{-1} \dot{+} B^{-1}\right)[g], g \in \mathcal{D}\left[A^{-1}\right] \cap \mathcal{D}\left[B^{-1}\right] .
\end{aligned}
$$

Therefore, $H_{\infty}^{-1}=A^{-1} \dot{+} B^{-1}, H_{\infty}=\left(A^{-1} \dot{+} B^{-1}\right)^{-1}=A$ : B, i.e., (4.2) holds true.

Theorem 4.3. Let $A$ be a nonnegative selfadjoint l.r. in $\mathcal{H}$ and let $\mathcal{L}$ be a subspace of $\mathcal{H}$. Then the following equality

$$
A_{\mathcal{L}}=\mathrm{s}-\mathrm{R}-\lim _{t \uparrow+\infty}\left(A: t P_{\mathcal{L}}\right)
$$

holds.

Proof. Set

$$
A(t):=A: t P_{\mathcal{L}}, t>0 .
$$

Then $A(t) \leq A$ for all $t>0, A(t)$ is a non-decreasing sequences of nonnegative selfadjoint l.r.,

$$
\operatorname{ran}(A(t))^{\frac{1}{2}}=\operatorname{ran} A^{\frac{1}{2}} \cap \mathcal{L} \forall t>0
$$

Hence $\operatorname{ran} A(t) \subseteq \mathcal{L}$ for all $t>0$ and, therefore, see (3.1), $A(t) \in \Xi(A, \mathcal{L})$ for all $t>0$. From [17. Theorem 3.1] it follows that there exists

$$
\mathrm{s}-\mathrm{R}-\lim _{t \uparrow+\infty} A(t)=: A_{0} .
$$

Since $\operatorname{ker} A(t) \supseteq \mathcal{L}^{\perp}$, we get ker $A_{0} \supseteq \mathcal{L}^{\perp} \Longleftrightarrow \operatorname{ran} A_{0} \subseteq \mathcal{L}$. Hence $A_{0} \in \Xi(A, \mathcal{L})$.

Let $\widetilde{A} \in \Xi(A, \mathcal{L})$. Set

$$
\widetilde{H}_{n}(t):=\left(\left(\widetilde{A}+\frac{1}{n} I\right)^{-1}+\left(t P+\frac{1}{n} I\right)^{-1}\right)^{-1}, n \in \mathbb{N}, t>0 .
$$

Because ker $\widetilde{A} \supseteq \mathcal{L}^{\perp}$ and ker $P_{\mathcal{L}}=\mathcal{L}^{\perp}, P_{\mathcal{L}} \uparrow \mathcal{L}=I_{\mathcal{L}}$, we have that

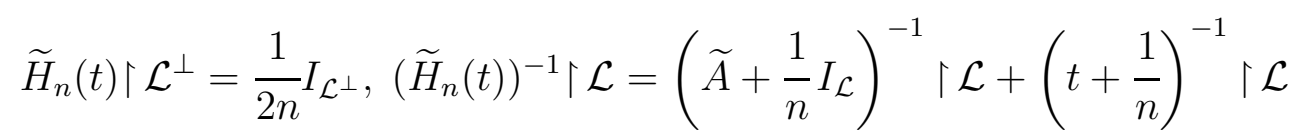

for all $n \in \mathbb{N}$ and for all $t>0$. Hence

$$
\mathrm{s}-\mathrm{R}-\lim _{n \rightarrow \infty}\left(\widetilde{H}_{n}(t)\right)^{-1} \uparrow \mathcal{L}=\widetilde{A}^{-1} \uparrow \mathcal{L}+t^{-1} I_{\mathcal{L}} \forall t>0 .
$$

Therefore,

$$
\widetilde{A}: t P_{\mathcal{L}}=\mathrm{s}-\mathrm{R}-\lim _{n \rightarrow \infty} \widetilde{H}_{n}(t)=\widetilde{A}
$$


Now the inequality $A \geq \widetilde{A}$ for all $\widetilde{A} \in \Xi(A, \mathcal{L})$ implies $A: t P_{\mathcal{L}} \geq \widetilde{A}: t P_{\mathcal{L}}$ for all $t>0$ and letting $t$ to $+\infty$ from (4.3) we get

$$
A_{0} \geq \widetilde{A} \forall \widetilde{A} \in \Xi(A, \mathcal{L})
$$

Due to Theorem 3.1, the shorted l.r. $A_{\mathcal{L}}$ is the maximal element of $\Xi(A, \mathcal{L})$. Therefore, $A_{0}=A_{\mathcal{L}}$. The proof is complete.

Corollary 4.4. Let $A$ and $B$ be nonnegative selfadjoint l.r. and let $\mathcal{L}$ be a subspace. Then

$$
(A: B)_{\mathcal{L}}=A_{\mathcal{L}}: B=A: B_{\mathcal{L}}=A_{\mathcal{L}}: B_{\mathcal{L}}
$$

Proof. By definition

$$
(A: B)_{\mathcal{L}}=\max \left\{C=C^{*}, C \geq 0: C \leq A: B, \operatorname{ran} C \subset \mathcal{L}\right\}
$$

Since $A_{\mathcal{L}}: B \leq A: B$ and

$$
\operatorname{ran}\left(A_{\mathcal{L}}: B\right)^{\frac{1}{2}}=\operatorname{ran}\left(A_{\mathcal{L}}\right)^{\frac{1}{2}} \cap \operatorname{ran} B^{\frac{1}{2}}=\mathcal{L} \cap \operatorname{ran} A^{\frac{1}{2}} \cap \operatorname{ran} B^{\frac{1}{2}} \subset \mathcal{L}
$$

we get that $(A: B)_{\mathcal{L}} \geq A_{\mathcal{L}}: B$ and similarly $(A: B)_{\mathcal{L}} \geq A: B_{\mathcal{L}}$.

On the other side because $B: t P_{\mathcal{L}}$ is a non-decreasing function w.r.t. $t>0$ and $B: t P_{\mathcal{L}} \leq$ $B_{\mathcal{L}}$, one has

$$
(A: B)_{\mathcal{L}}=\mathrm{s}-\mathrm{R}-\lim _{t \uparrow+\infty}\left((A: B): t P_{\mathcal{L}}\right)=\mathrm{s}-\mathrm{R}-\lim _{t \uparrow+\infty}\left(A:\left(B: t P_{\mathcal{L}}\right)\right) \leq A: B_{\mathcal{L}}
$$

Hence

$$
A: B_{\mathcal{L}} \geq(A: B)_{\mathcal{L}} \geq A: B_{\mathcal{L}} \Longleftrightarrow(A: B)_{\mathcal{L}}=A: B_{\mathcal{L}}
$$

and similarly $(A: B)_{\mathcal{L}}=A_{\mathcal{L}}: B$.

Since $A_{\mathcal{L}} \leq A$, we get $A_{\mathcal{L}}: B_{\mathcal{L}} \leq A: B_{\mathcal{L}}=(A: B)_{\mathcal{L}}$. On the other side

$$
A_{\mathcal{L}} \geq A: t P_{\mathcal{L}} \Longrightarrow A_{\mathcal{L}}: B_{\mathcal{L}} \geq\left(A: t P_{\mathcal{L}}\right): B_{\mathcal{L}}=\left(A: B_{\mathcal{L}}\right): t P_{\mathcal{L}} \forall t>0
$$

Hence

$$
A_{\mathcal{L}}: B_{\mathcal{L}} \geq \mathrm{s}-\mathrm{R}-\lim _{t \uparrow+\infty}\left(\left(A: B_{\mathcal{L}}\right): t P_{\mathcal{L}}\right)=\left(A: B_{\mathcal{L}}\right)_{\mathcal{L}}=A: B_{\mathcal{L}}=(A: B)_{\mathcal{L}}
$$

\section{The inequality BetWeen The haRmoniC AND ARITHMEtiC MEANS OF NONNEGATIVE SELFADJOINT LINEAR RELATIONS}

If $a$ and $b$ are positive numbers, then the harmonic mean and the arithmetic mean of $a$ and $b$ are connected by the inequality

$$
h(a, b)=\left(\frac{a^{-1}+b^{-1}}{2}\right)^{-1} \leq \frac{a+b}{2},
$$

and the equality holds if and only if $a=b$.

Let $A, B \in \mathbf{B}^{+}(\mathcal{H})$ and let $\mathfrak{L}:=\overline{\operatorname{ran}}(A+B)$, then since $A+B \geq A, B$, there exists a nonnegative selfadjoint contraction $K$ in $\mathfrak{L}$ such that

$$
A=(A+B)^{\frac{1}{2}} K(A+B)^{\frac{1}{2}}, B=(A+B)^{\frac{1}{2}}\left(I_{\mathfrak{L}}-K\right)(A+B)^{\frac{1}{2}} .
$$

Actually, since $\left\|(A+B)^{\frac{1}{2}} f\right\|^{2} \geq\left\|A^{\frac{1}{2}} f\right\|^{2}$ for all $f \in \mathcal{H}$, by the Douglas factorization theorem 23. there exists a contraction $X \in \mathbf{B}(\mathcal{H}, \mathfrak{L})$ such that $A^{\frac{1}{2}}=(A+B)^{\frac{1}{2}} X$. Then $A=$ $(A+B)^{\frac{1}{2}} X X^{*}(A+B)^{\frac{1}{2}}$. The following equality is established in [10]:

$$
h(A, B)=2(A+B)^{\frac{1}{2}}\left(I_{\mathfrak{L}}-K\right) K(A+B)^{\frac{1}{2}} .
$$


It follows that

$$
h(A, B) \leq \frac{A+B}{2}
$$

and the equality holds if and only if $A=B$.

The goal of this section is to prove a similar inequality for the case of nonnegative selfadjoint l.r.. First, we find the Cayley transforms of the arithmetic and harmonic means.

Lemma 5.1. Let $M$ be a nonnegative selfadjoint contraction in the Hilbert space $\mathfrak{L}$. Then

$$
\left(M-M^{2}\right)^{-1}[f]=M^{-1}[f]+(I-M)^{-1}[f] \forall f \in \operatorname{ran}\left(M-M^{2}\right)^{\frac{1}{2}} .
$$

Proof. It is sufficient to consider the case $\operatorname{ker} M=\operatorname{ker}(I-M)=\{0\}$. Then for $f=$ $\left(M-M^{2}\right)^{\frac{1}{2}} u$ we get

$$
M^{-\frac{1}{2}} f=(I-M)^{\frac{1}{2}} u,(I-M)^{-\frac{1}{2}} f=M^{\frac{1}{2}} u,\left(M-M^{2}\right)^{-\frac{1}{2}} f=u .
$$

Hence

$$
\begin{aligned}
& \left\|\left(M-M^{2}\right)^{-\frac{1}{2}} f\right\|^{2}=\|u\|^{2},\left\|M^{-\frac{1}{2}} f\right\|^{2}=\left\|(I-M)^{\frac{1}{2}} u\right\|^{2}=\|u\|^{2}-\left\|M^{\frac{1}{2}} u\right\|^{2}, \\
& \left\|(I-M)^{-\frac{1}{2}} f\right\|^{2}=\left\|M^{\frac{1}{2}} u\right\|^{2} .
\end{aligned}
$$

This completes the proof.

Theorem 5.2. Let $A$ and $B$ be nonnegative selfadjoint l.r. and let

$$
T_{A}:=\mathfrak{C}(A), T_{B}:=\mathfrak{C}(B)
$$

be the Cayley transforms of $A$ and $B$, respectively. Then

$$
\begin{gathered}
\mathfrak{C}\left(\frac{1}{2}(A \dot{+} B)\right)=h\left(I+T_{A}, I+T_{B}\right)-I, \\
\mathfrak{C}(h(A, B))=I-h\left(I-T_{A}, I-T_{B}\right) .
\end{gathered}
$$

Proof. Set

$$
\begin{aligned}
& \widetilde{S}:=\frac{1}{2}(A \dot{+} B), L_{A}=I+T_{A}, L_{B}=I+T_{B}, \\
& \widetilde{T}=h\left(I+T_{A}, I+T_{B}\right)-I, \widetilde{L}=I+\widetilde{T} .
\end{aligned}
$$

Since

$$
\widetilde{T} \geq-I, \widetilde{T} \leq \frac{1}{2}\left(I+T_{A}+I+T_{B}\right)-I=\frac{1}{2}\left(T_{A}+T_{B}\right) \leq I,
$$

the operator $\widetilde{T}$ is a selfadjoint contraction. Using (5.1) we get the representation

$$
h\left(I+T_{A}, I+T_{B}\right)=I+\widetilde{L}=2\left(L_{A}+L_{B}\right)^{\frac{1}{2}}\left(M-M^{2}\right)\left(L_{A}+L_{B}\right)^{\frac{1}{2}},
$$

where $M$ is a nonnegative selfadjoint contraction acting in the subspace $\overline{\operatorname{ran}}\left(L_{A}+L_{B}\right)$ and such that

$$
L_{A}=\left(L_{A}+L_{B}\right)^{\frac{1}{2}} M\left(L_{A}+L_{B}\right)^{\frac{1}{2}}, L_{B}=\left(L_{A}+L_{B}\right)^{\frac{1}{2}}(I-M)\left(L_{A}+L_{B}\right)^{\frac{1}{2}} .
$$

From (2.7) we have

$$
\begin{gathered}
\mathcal{D}[A]=\operatorname{ran} L_{A}^{\frac{1}{2}}, \quad A[v]+\|v\|^{2}=2 L_{A}^{-1}[v], v \in \mathcal{D}[A], \\
\mathcal{D}[B]=\operatorname{ran} L_{B}^{\frac{1}{2}}, \quad B[u]+\|u\|^{2}=2 L_{B}^{-1}[u], u \in \mathcal{D}[B], \\
\mathcal{D}[\widetilde{S}]=\operatorname{ran} L_{A}^{\frac{1}{2}} \cap \operatorname{ran} L_{B}^{\frac{1}{2}}, \widetilde{S}[f]+\|f\|^{2}=L_{A}^{-1}[f]+L_{B}^{-1}[f], f \in \mathcal{D}[\widetilde{S}] .
\end{gathered}
$$


From (5.5) one gets

$$
\left\{\begin{array}{l}
\operatorname{ran} L_{A}^{\frac{1}{2}}=\left(L_{A}+L_{B}\right)^{\frac{1}{2}} \operatorname{ran} M^{\frac{1}{2}} \\
L_{A}^{-1}[v]=\left\|M^{\left[-\frac{1}{2}\right]}\left(L_{A}+L_{B}\right)^{\left[-\frac{1}{2}\right]} v\right\|^{2}, v \in \operatorname{ran} L_{A}^{\frac{1}{2}} \\
\operatorname{ran} L_{B}^{\frac{1}{2}}=\left(L_{A}+L_{B}\right)^{\frac{1}{2}} \operatorname{ran}(I-M)^{\frac{1}{2}}, \\
L_{B}^{-1}[u]=\left\|(I-M)^{\left[-\frac{1}{2}\right]}\left(L_{A}+L_{B}\right)^{\left[-\frac{1}{2}\right]} u\right\|^{2}, u \in \operatorname{ran} L_{B}^{\frac{1}{2}}
\end{array}\right.
$$

Hence

$$
\left\{\begin{array}{l}
\mathcal{D}[\widetilde{S}]=\left(L_{A}+L_{B}\right)^{\frac{1}{2}}\left(\operatorname{ran} M^{\frac{1}{2}} \cap \operatorname{ran}(I-M)^{\frac{1}{2}}\right)=\left(L_{A}+L_{B}\right)^{\frac{1}{2}}\left(\operatorname{ran}\left(M-M^{2}\right)^{\frac{1}{2}}\right), \\
\widetilde{S}[f]+\|f\|^{2}=\left\|M^{\left[-\frac{1}{2}\right]}\left(L_{A}+L_{B}\right)^{\left[-\frac{1}{2}\right]} f\right\|^{2}+\left\|(I-M)^{\left[-\frac{1}{2}\right]}\left(L_{A}+L_{B}\right)^{\left[-\frac{1}{2}\right]} f\right\|^{2}, f \in \mathcal{D}[\widetilde{S}]
\end{array} .\right.
$$

Now Lemma 5.1 and (5.4) yield that

$$
\left\{\begin{array}{l}
\mathcal{D}[\widetilde{S}]=\operatorname{ran}(I+\widetilde{L})^{\frac{1}{2}} \\
\widetilde{S}[f]+\|f\|^{2}=\left(M-M^{2}\right)^{-1}\left[\left(L_{A}+L_{B}\right)^{\left[-\frac{1}{2}\right]} f\right]=2\left\|(I+\widetilde{L})^{\left[-\frac{1}{2}\right]} f\right\|^{2}, f \in \mathcal{D}[\widetilde{S}]
\end{array} .\right.
$$

Taking into account (2.7) we obtain that $\mathfrak{C}(\widetilde{S})=\widetilde{L}$.

Since

we get

$$
\mathfrak{C}\left(A^{-1}\right)=-T_{A}, \mathfrak{C}\left(B^{-1}\right)=-T_{B}
$$

$$
\mathfrak{C}\left(\frac{1}{2}\left(A^{-1} \dot{+} B^{-1}\right)\right)=h\left(I-T_{A}, I-T_{B}\right)-I .
$$

Then

$$
\mathfrak{C}(h(A, B))=\mathfrak{C}\left(\left(\frac{A^{-1} \dot{+} B^{-1}}{2}\right)^{-1}\right)=I-h\left(I-T_{A}, I-T_{B}\right) .
$$

Now from (1.12), (2.7) and (5.3) we get the equalities

$$
\begin{aligned}
h(A, B)= & \{\{(2 I-h(I-\mathfrak{C}(A), I-\mathfrak{C}(B))) f,(h(I-\mathfrak{C}(A), I-\mathfrak{C}(B))) f\}, f \in \mathcal{H}\} \\
& \mathcal{D}[A: B]=\mathcal{D}[h(A, B)]=\operatorname{ran}(2 I-h(I-\mathfrak{C}(A), I-\mathfrak{C}(B)))^{\frac{1}{2}} .
\end{aligned}
$$

The next statement is an analogue of [44, Theorem 2.5].

Corollary 5.3. Let $\left\{A_{n}\right\}$ and $\left\{B_{n}\right\}$ be two sequences of nonnegative selfadjoint l.r.. Then in the strong resolvent convergence sense

$$
\begin{gathered}
A_{n} \nearrow A_{\infty}, B_{n} \nearrow B_{\infty} \Longrightarrow A_{n} \dot{+} B_{n} \nearrow A_{\infty} \dot{+} B_{\infty}, \\
A_{n} \searrow A_{\infty}, B_{n} \searrow B_{\infty} \Longrightarrow\left(A_{n}: B_{n}\right) \searrow\left(A_{\infty}: B_{\infty}\right) .
\end{gathered}
$$

Proof. Set $T_{A_{n}}:=\mathfrak{C}\left(A_{n}\right), T_{B_{n}}:=\mathfrak{C}\left(B_{n}\right), n \in \mathbb{N}, T_{A_{\infty}}:=\mathfrak{C}\left(A_{\infty}\right), T_{B_{\infty}}:=\mathfrak{C}\left(B_{\infty}\right)$.

If $A_{n} \nearrow A_{\infty}$ and $B_{n} \nearrow B_{\infty}$ in the strong resolvent sense, then $T_{A_{n}} \searrow T_{A_{\infty}}$ and $T_{B_{n}} \searrow T_{B_{\infty}}$ in the strong sense. It follows from (5.2) and [44, Theorem 2.5.] that

$$
\begin{aligned}
& \mathfrak{C}\left(\frac{1}{2}\left(A_{n} \dot{+} B_{n}\right)\right)=h\left(I+T_{A_{n}}, I+T_{B_{n}}\right)-I \\
& \quad=2\left(I+T_{A_{n}}: I+T_{B_{n}}\right)-I \searrow 2\left(I+T_{A_{\infty}}: I+T_{B_{\infty}}\right)-I=h\left(I+T_{A_{\infty}}, I+T_{B_{\infty}}\right)-I .
\end{aligned}
$$


Now

$$
\frac{1}{2}\left(A_{\infty} \dot{+} B_{\infty}\right)=\mathfrak{C}\left(h\left(I+T_{A_{\infty}}, I+T_{B_{\infty}}\right)-I\right)=\mathrm{s}-\mathrm{R}-\lim _{n \rightarrow \infty} \frac{1}{2}\left(A_{n} \dot{+} B_{n}\right) .
$$

Similarly, if $A_{n} \searrow A_{\infty}$ and $B_{n} \searrow B_{\infty}$, then, taking into account equality [5.3] and [44, Theorem 2.5.] once again, we get that $\left(A_{n}: B_{n}\right) \searrow\left(A_{\infty}: B_{\infty}\right)$.

For nonnegative selfadjoint l.r. $A$ and $B$ let us define a nonnegative selfadjoint l.r. $c_{0}(A, B)$ as follows

$$
\begin{aligned}
c_{0}(A, B):=\mathfrak{C}\left(\frac{\mathfrak{C}(A)+\mathfrak{C}(B)}{2}\right) \\
\quad=\left\{\left\{\left(I+\frac{\mathfrak{C}(A)+\mathfrak{C}(B)}{2}\right) f,\left(I-\frac{\mathfrak{C}(A)+\mathfrak{C}(B)}{2}\right) f\right\}: f \in \mathcal{H}\right\} .
\end{aligned}
$$

If $a$ and $b$ are positive numbers, then

$$
c_{0}(a, b)=\frac{a+b+2 a b}{2+a+b} .
$$

Observe that the following inequalities are valid

$$
\frac{2 a b}{a+b} \leq \frac{a+b+2 a b}{2+a+b} \leq \frac{a+b}{2}
$$

and the sign " = " holds if and only if $a=b$.

Theorem 5.4. Let $A$ and $B$ be a nonnegative selfadjoint l.r.. Then

(1) $\mathcal{D}\left[c_{0}(A, B)\right]=\mathcal{D}[A]+\mathcal{D}[B]$

(2) $\operatorname{ran}\left(c_{0}(A, B)\right)^{\frac{1}{2}}=\operatorname{ran} A^{\frac{1}{2}}+\operatorname{ran} B^{\frac{1}{2}}$;

(3) the inclusion

$$
\mathcal{D}[A: B] \supseteq \mathcal{D}[A]+\mathcal{D}[B]
$$

holds and the inequality

$$
h(A, B) \leq c_{0}(A, B) \leq \frac{A \dot{+} B}{2}
$$

In addidion

is valid; moreover, the sign "=" holds if and only if $A=B$.

$$
\operatorname{ran}(A \dot{+} B)^{\frac{1}{2}} \supseteq \operatorname{ran} A^{\frac{1}{2}}+\operatorname{ran} B^{\frac{1}{2}}
$$

Proof. Set

$$
T_{A}:=\mathfrak{C}(A), T_{B}:=\mathfrak{C}(B) .
$$

Because $I \pm T_{A}$ and $I \pm T_{B}$ are bounded nonnegative operators, we have the inequalities

$$
h\left(I \pm T_{A}, I \pm T_{B}\right) \leq \frac{1}{2}\left(I \pm T_{A}+I \pm T_{B}\right)=I \pm \frac{1}{2}\left(T_{A}+T_{B}\right) .
$$

Using (15.2), (15.3), and (5.7) we get

$$
\begin{aligned}
h\left(I+T_{A}, I+T_{B}\right)-I & \leq \frac{1}{2}\left(T_{A}+T_{B}\right) \leq I-h\left(I-T_{A}, I-T_{B}\right) \\
\Longleftrightarrow \mathfrak{C}\left(\frac{1}{2}(A \dot{+} B)\right) & \leq \mathfrak{C}\left(c_{0}(A, B)\right) \leq \mathfrak{C}(h(A, B)) \Longleftrightarrow h(A, B) \leq c_{0}(A, B) \leq \frac{1}{2}(A \dot{+} B) .
\end{aligned}
$$


If $A=B$, then, clearly,

$$
c_{0}(A, B)=A, \frac{1}{2}(A \dot{+} B)=A, \frac{1}{2}\left(A^{-1} \dot{+} B^{-1}\right)=A^{-1} \Longrightarrow h(A, B)=A .
$$

Thus, $h(A, B)=c_{0}(A, B)=\frac{1}{2}(A \dot{+} B)$.

Suppose that $h(A, B)=\frac{1}{2}(A \dot{+} B)$ for some nonnegative selfadjoint l.r. $A$ and $B$. Then

$$
I-h\left(I-T_{A}, I-T_{B}\right)=h\left(I+T_{A}, I+T_{B}\right)-I .
$$

Since

$$
\frac{1}{2}\left(T_{A}+T_{B}\right) \leq I-h\left(I-T_{A}, I-T_{B}\right)=h\left(I+T_{A}, I+T_{B}\right)-I \leq \frac{1}{2}\left(T_{A}+T_{B}\right),
$$

we get

$$
h\left(I+T_{A}, I+T_{B}\right)-I=\frac{1}{2}\left(T_{A}+T_{B}\right) \Longleftrightarrow h\left(I+T_{A}, I+T_{B}\right)=\frac{1}{2}\left(I+T_{A}+I+T_{B}\right) .
$$

Because $I+T_{A}$ and $I+T_{B}$ are bounded, the latter equality yields the equality $T_{A}=T_{B}$ and hence

$$
A=\mathfrak{C}\left(T_{A}\right)=\mathfrak{C}\left(T_{B}\right)=B .
$$

Similarly, one can prove that $h(A, B)=c_{0}(A, B) \Longrightarrow A=B$, and $c_{0}(A, B)=\frac{1}{2}(A \dot{+} B) \Longrightarrow$ $A=B$.

From (2.7) and (5.3) we have

$$
\begin{aligned}
& \mathcal{D}[A: B]=\mathcal{D}[h(A, B)]=\operatorname{ran}\left(2 I-h\left(I-T_{A}, I-T_{B}\right)\right)^{\frac{1}{2}}, \\
& \mathcal{D}\left[c_{0}(A, B)\right]=\operatorname{ran}\left(\frac{I+T_{A}}{2}+\frac{I+T_{B}}{2}\right)^{\frac{1}{2}}, \operatorname{ran}\left(c_{0}(A, B)\right)^{\frac{1}{2}}=\operatorname{ran}\left(\frac{I-T_{A}}{2}+\frac{I-T_{B}}{2}\right)^{\frac{1}{2}} .
\end{aligned}
$$

Since

we obtain the inequality

$$
h\left(I \pm T_{A}, I \pm T_{B}\right) \leq \frac{\left(I \pm T_{A}\right)+\left(I \pm T_{B}\right)}{2}=I \pm \frac{T_{A}+T_{B}}{2},
$$

$$
2 I-h\left(I \pm T_{A}, I \pm T_{B}\right) \geq \frac{I \mp T_{A}}{2}+\frac{I \mp T_{B}}{2} .
$$

Now the Douglas theorem [23] yields the inclusions

$$
\operatorname{ran}\left(2 I-h\left(I \pm T_{A}, I \pm T_{B}\right)\right)^{\frac{1}{2}} \supseteq \operatorname{ran}\left(\frac{I \mp T_{A}}{2}+\frac{I \mp T_{B}}{2}\right)^{\frac{1}{2}} .
$$

But, see [26, Theorem 2.2],

$$
\operatorname{ran}\left(\frac{I \pm T_{A}}{2}+\frac{I \pm T_{B}}{2}\right)^{\frac{1}{2}}=\operatorname{ran}\left(I \pm T_{A}\right)^{\frac{1}{2}}+\operatorname{ran}\left(I \pm T_{B}\right)^{\frac{1}{2}} .
$$

Using (5.6) and equalities

$$
\begin{aligned}
\mathcal{D}[A]=\operatorname{ran}\left(I+T_{A}\right)^{\frac{1}{2}}, \mathcal{D}[B]=\operatorname{ran}\left(I+T_{B}\right)^{\frac{1}{2}}, & \\
& \operatorname{ran} A^{\frac{1}{2}}=\operatorname{ran}\left(I-T_{A}\right)^{\frac{1}{2}}, \operatorname{ran} B^{\frac{1}{2}}=\operatorname{ran}\left(I-T_{A}\right)^{\frac{1}{2}}
\end{aligned}
$$

we get the equalities

$$
\mathcal{D}\left[c_{0}(A, B)\right]=\mathcal{D}[A]+\mathcal{D}[B], \operatorname{ran}\left(c_{0}(A, B)\right)^{\frac{1}{2}}=\operatorname{ran} A^{\frac{1}{2}}+\operatorname{ran} B^{\frac{1}{2}},
$$


and finally arrive at (5.9) . Since

$$
\operatorname{ran}\left(2 I-h\left(I+T_{A}, I+T_{B}\right)\right)^{\frac{1}{2}} \supseteq \operatorname{ran}\left(\left(I-T_{A}\right)+\left(I-T_{B}\right)\right)^{\frac{1}{2}},
$$

we get (5.10).

The proof is complete.

As a consequence of (5.9) we note, that if one of two nonnegative selfadjoint l.r. is a linear operator, then their parallel sum is a nonnegative selfadjoint linear operator as well, moreover, if one of two is a bounded operator, then the parallel sum is a bounded operator.

\section{Arithmetic-HARmonic Means}

We consider analogs of the arithmetic-harmonic means for the case of nonnegative selfadjoint l.r..

Theorem 6.1. Let $A$ and $B$ be nonnegative selfadjoint l.r.. Define

$$
A_{0}:=A, B_{0}:=B, A_{n}:=\frac{1}{2}\left(A_{n-1} \dot{+} B_{n-1}\right), B_{n}:=h\left(A_{n-1}, B_{n-1}\right), n \in \mathbb{N} .
$$

Then the sequence $\left\{A_{n}\right\}$ is a non-increasing, while the sequence $\left\{B_{n}\right\}$ is a non-decreasing and $A_{n} \geq B_{n}$ for all $n \in \mathbb{N}$. Moreover, the nonnegative selfadjoint l.r.

$$
A_{\infty}:=\mathrm{s}-\mathrm{R}-\lim _{n \rightarrow \infty} A_{n}, B_{\infty}:=\mathrm{s}-\mathrm{R}-\lim _{n \rightarrow \infty} B_{n}
$$

posses the following properties:

$$
\begin{aligned}
& h(A, B) \leq B_{\infty} \leq A_{\infty} \leq \frac{1}{2}(A \dot{+} B), \\
& \mathcal{D}\left[B_{\infty}\right] \supseteq \mathcal{D}\left[A_{\infty}\right] \supseteq \mathcal{D}[A] \cap \mathcal{D}[B], \\
& \operatorname{ran} A_{\infty}^{\frac{1}{2}} \supseteq \operatorname{ran} B_{\infty}^{\frac{1}{2}} \supseteq \operatorname{ran} A^{\frac{1}{2}} \cap \operatorname{ran} B^{\frac{1}{2}}, \\
& A_{\infty}[\varphi]=B_{\infty}[\varphi] \forall \varphi \in \mathcal{D}\left[A_{\infty}\right], \\
& A_{\infty}^{-1}[\psi]=B_{\infty}^{-1}[\psi] \forall \psi \in \operatorname{ran} B_{\infty}^{\frac{1}{2}} .
\end{aligned}
$$

If $A^{-1}$ and $B^{-1}$ are bounded (i.e. they are graphs of bounded nonnegative selfadjoint operators), then

$$
A_{\infty}=B_{\infty}=\left(A^{-1} \# B^{-1}\right)^{-1} .
$$

Proof. By induction from the definitions of form sums, harmonic means and from Theorem 5.4 one can show that

$$
\begin{aligned}
& \mathcal{D}\left[A_{n}\right]=\mathcal{D}[A] \cap \mathcal{D}[B], \mathcal{D}\left[B_{n}\right] \supseteq \mathcal{D}[A]+\mathcal{D}[B] \\
& h(A, B)=B_{1} \leq B_{n} \leq B_{n+1} \leq A_{n+1} \leq A_{n} \leq A_{1}=\frac{1}{2}(A \dot{+} B) \quad \forall n \in \mathbb{N} .
\end{aligned}
$$

Since the sequence $\left\{B_{n}\right\}$ is a non-decreasing and the sequence $\left\{A_{n}\right\}$ is a non-increasing, from [33, Theorem VII.3.11], [49, Theorem 3.1, Theorem 3.2], [17, Theorem 4.2, Theorem 4.3] it follows that the strong resolvent limits of $\left\{A_{n}\right\}$ and $\left\{B_{n}\right\}$ exist and

$$
h(A, B) \leq B_{\infty} \leq A_{\infty} \leq \frac{1}{2}(A \dot{+} B), \mathcal{D}\left[B_{\infty}\right] \supseteq \mathcal{D}\left[A_{\infty}\right] .
$$

Since

$$
\mathcal{D}\left[B_{\infty}\right]=\left\{h \in \bigcap_{n=1}^{\infty} \mathcal{D}\left[B_{n}\right]: \lim _{n \rightarrow \infty} B_{n}[h]<\infty\right\}
$$


and $B_{n} \leq A_{1}$ for all $n \in \mathbb{N}, \bigcap_{n=1}^{\infty} \mathcal{D}\left[B_{n}\right] \supseteq \mathcal{D}[A] \cap \mathcal{D}[B]=\mathcal{D}\left[A_{1}\right]$, we get that

$$
\mathcal{D}\left[B_{\infty}\right] \supseteq \mathcal{D}[A] \cap \mathcal{D}[B]
$$

Fix $n \in \mathbb{N}$. Then for any $f \in \mathcal{D}[A] \cap \mathcal{D}[B]$ by definition and from $B_{n+1} \geq B_{n}$ we have

$$
\begin{aligned}
A_{n+1}[f]-B_{n+1}[f]=\frac{1}{2} A_{n}[f]+\frac{1}{2} B_{n}[f]- & B_{n+1}[f] \\
& \leq \frac{1}{2} A_{n}[f]+\frac{1}{2} B_{n}[f]-B_{n}[f] \leq \frac{A_{n}[f]-B_{n}[f]}{2} .
\end{aligned}
$$

Hence

$$
0 \leq A_{n+1}[f]-B_{n+1}[f] \leq \frac{A_{1}[f]-B_{1}[f]}{2^{n}} \forall f \in \mathcal{D}[A] \cap \mathcal{D}[B], \forall n \in \mathbb{N} .
$$

It follows that

$$
\lim _{n \rightarrow \infty} A_{n}[f]=\lim _{n \rightarrow \infty} B_{n}[f]=B_{\infty}[f] \quad \forall f \in \mathcal{D}[A] \cap \mathcal{D}[B] .
$$

Define the sesquilinear form $\mathfrak{t}$ as follows

$$
\operatorname{dom} \mathfrak{t}=\bigcup_{n=1}^{\infty} \mathcal{D}\left[A_{n}\right], \mathfrak{t}[f, g]=\lim _{n \rightarrow \infty} A_{n}[f, g], f, g \in \operatorname{dom} \mathfrak{t}
$$

Since $\mathcal{D}\left[A_{n}\right]=\mathcal{D}[A] \cap \mathcal{D}[B]$, we have the equality dom $\mathfrak{t}=\mathcal{D}[A] \cap \mathcal{D}[B]$ and from (6.3):

$$
\mathfrak{t}[f, g]=B_{\infty}[f, g] \quad \forall f, g, \in \mathcal{D}[A] \cap \mathcal{D}[B] .
$$

Since the form $B_{\infty}[\cdot, \cdot]$ restricted on $\mathcal{D}[A] \cap \mathcal{D}[B]$ is closable, using [49, Theorem 3.2], [17, Theorem 4.3], we obtain that the closure of the form $\mathfrak{t}[\cdot, \cdot]$ coincides with $A_{\infty}[\cdot, \cdot]$. Hence $A_{\infty}[\varphi]=B_{\infty}[\varphi]$ for all $\varphi \in \mathcal{D}\left[A_{\infty}\right]$.

Since

$$
\begin{aligned}
& A_{n}^{-1}=2\left(A_{n-1} \dot{+} B_{n-1}\right)^{-1}=h\left(A_{n-1}^{-1}, B_{n-1}^{-1}\right), \\
& B_{n}^{-1}=\left(h\left(A_{n-1}, B_{n-1}\right)\right)^{-1}=\frac{1}{2}\left(A_{n-1}^{-1} \dot{+} B_{n-1}^{-1}\right), n \in \mathbb{N},
\end{aligned}
$$

the sequences $\left\{A_{n}^{-1}\right\},\left\{B_{n}^{-1}\right\} \subset \mathbf{B}^{+}(\mathcal{H})$ are the non-decreasing and non-increasing, respectively, and

$$
\mathrm{s}-\mathrm{R}-\lim _{n \rightarrow \infty} A_{n}^{-1}=A_{\infty}^{-1}, \mathrm{~s}-\mathrm{R}-\lim _{n \rightarrow \infty} B_{n}^{-1}=B_{\infty}^{-1} .
$$

Arguing as above we see that

$$
B_{\infty}^{-1} \geq A_{\infty}^{-1}, \operatorname{ran} A_{\infty}^{\frac{1}{2}} \supseteq \operatorname{ran} B_{\infty}^{\frac{1}{2}} \supseteq \operatorname{ran} A^{\frac{1}{2}} \cap \operatorname{ran} B^{\frac{1}{2}}
$$

and

$$
A_{\infty}^{-1}[\psi]=B_{\infty}^{-1}[\psi] \quad \forall \psi \in \operatorname{ran} B_{\infty}^{\frac{1}{2}}
$$

Suppose that $A_{0}^{-1}=A^{-1}$ and $B_{0}^{-1}=B^{-1}$ are bounded. Then from (4.1) it follows that $\left\{A_{n}^{-1}\right\},\left\{B_{n}^{-1}\right\} \subset \mathbf{B}^{+}(\mathcal{H})$, and there is a common limit

$$
\mathrm{s}-\lim _{n \rightarrow \infty} A_{n}^{-1}=\mathrm{s}-\lim _{n \rightarrow \infty} B_{n}^{-1}=A^{-1} \# B^{-1} .
$$

Due to the equalities

$$
A_{\infty}=\left(\mathrm{s}-\lim _{n \rightarrow \infty} A_{n}^{-1}\right)^{-1}, B_{\infty}=\left(\mathrm{s}-\lim _{n \rightarrow \infty} B_{n}^{-1}\right)^{-1}
$$

we get $A_{\infty}=B_{\infty}=\left(A^{-1} \# B^{-1}\right)^{-1}$. The proof is complete. 
In the sequel we will denote by $a h(A, B)$ the arithmetic-harmonic means of nonnegative selfadjoint l.r. $A$ and $B$. By Theorem 6.1 we have

$$
\operatorname{ah}(A, B)=\left\langle A_{\infty}, B_{\infty}\right\rangle
$$

where $A_{\infty}$ and $B_{\infty}$ are defined in (6.1) and (6.2). Then from (4.1)

$$
\operatorname{ah}\left(A^{-1}, B^{-1}\right)=\left\langle B_{\infty}^{-1}, A_{\infty}^{-1}\right\rangle .
$$

The next corollaries, show that in general $A_{\infty} \neq B_{\infty}$.

Corollary 6.2. Let $B$ be a nonnegative selfadjoint l.r.. Define

$$
\begin{aligned}
& \mathcal{B}^{\prime}:=\{\overline{\operatorname{dom}} B \oplus\{0\}\} \bigoplus\{\{0\} \oplus \operatorname{mul} B\}=\{\{\varphi, \psi\}: \varphi \in \overline{\operatorname{dom}} B, \psi \in \operatorname{mul} B\} \\
& \mathcal{B}^{\prime \prime}:=\{\operatorname{ker} B \oplus\{0\}\} \bigoplus\{\{0\} \oplus \overline{\operatorname{ran}} B\}=\{\{f, g\}: f \in \operatorname{ker} B, g \in \overline{\operatorname{ran}} B\} .
\end{aligned}
$$

Then

(1) for $A=0(\operatorname{dom} A=\mathcal{H})$ one has ah $(A, B)=\left\langle\mathcal{B}^{\prime}, A\right\rangle$,

(2) for $A=\{0\} \oplus \mathcal{H}$ one has ah $(A, B)=\left\langle A, \mathcal{B}^{\prime \prime}\right\rangle$.

Proof. (1) If $A$ is zero operator, defined on $\mathcal{H}$, then for sequences $\left\{A_{n}\right\}$ and $\left\{B_{n}\right\}$, defined in (6.1), we have $A_{1}:=\frac{1}{2}(A \dot{+} B)=\frac{1}{2} B, B_{1}:=h(A, B)=A$,

$$
A_{n}=\frac{1}{2^{n}} B, B_{n}=A, n \in \mathbb{N}
$$

Thus $A_{\infty}=\mathcal{B}^{\prime}, B_{\infty}=A$.

(2) If $A=\{0\} \oplus \mathcal{H}$, then $A^{-1}$ is the zero operator defined on $\mathcal{H}$. Hence we can use (4.1) and the arguments above.

Corollary 6.3. Let $A$ be a nonnegative selfadjoint l.r.. Set

$$
\mathfrak{M}:=\operatorname{mul} A \oplus \operatorname{ker} A \text {. }
$$

Then $\operatorname{ah}\left(A, A^{-1}\right)=\left\langle\left(P_{\mathfrak{M}^{\perp}}\right)^{-1}, P_{\mathfrak{M}^{\perp}}\right\rangle$. In particular ah $\left(A, A^{-1}\right)=I \Longleftrightarrow \mathfrak{M}=\{0\}$.

Proof. Set $T:=\mathfrak{C}(A)$, then $\mathfrak{C}\left(A^{-1}\right)=-T$,

$$
A_{1}:=\frac{1}{2}\left(A \dot{+} A^{-1}\right), B_{1}:=h\left(A, A^{-1}\right)=\left(\frac{1}{2}\left(A^{-1} \dot{+} A\right)\right)^{-1}=A_{1}^{-1} \text {. }
$$

By induction

$$
A_{n}:=\frac{1}{2}\left(A_{n-1} \dot{+} B_{n-1}\right)=\frac{1}{2}\left(A_{n-1} \dot{+} A_{n-1}^{-1}\right), B_{n}:=h\left(A_{n-1}, B_{n-1}\right)=A_{n}^{-1}, n \geq 2 .
$$

Using (5.2) and (5.3) we get

$$
\begin{aligned}
& \mathfrak{C}\left(A_{1}\right)=h(I+T, I-T)-I=2(I+T)(I-T)(I+T+I-T)^{-1}-I=-T^{2}, \\
& \mathfrak{C}\left(B_{1}\right)=\mathfrak{C}\left(A_{1}^{-1}\right)=-\mathfrak{C}\left(A_{1}\right)=T^{2}, \mathfrak{C}\left(A_{n}\right)=-T^{2^{n}}, \mathfrak{C}\left(B_{n}\right)=T^{2^{n}}, n \geq 2 .
\end{aligned}
$$

Since $T$ is a selfadjoint contraction and $\mathfrak{M}=\operatorname{ker}\left(I-T^{2}\right)$, where $\mathfrak{M}$ is defined by (6.4), we get the equality

$$
\mathrm{s}-\lim _{n \rightarrow \infty} T^{2^{n}}=P_{\mathfrak{M}}
$$

It follows that

$$
B_{\infty}=\mathfrak{C}\left(P_{\mathfrak{M}}\right)=\left(I-P_{\mathfrak{M}}\right)\left(I+P_{\mathfrak{M}}\right)^{-1}=P_{\mathfrak{M}^{\perp}}, A_{\infty}=\mathfrak{C}\left(-P_{\mathfrak{M}}\right)=\left(P_{\mathfrak{M}^{\perp}}\right)^{-1}
$$

The proof is complete. 


\section{Arithmetic, harmonic, ARITHMEtiC-HARMonic MeAns AND NONNEGATIVE SELFADJOINT EXTENSIONS OF NONNEGATIVE SYMMETRIC LINEAR RELATIONS}

Basic results of the Krein theory [34] can be extended to the case of a non-densely defined symmetric operator or a symmetric l.r. [7, 11, 18, 28, 29, 30]. Let $S$ be a nonnegative symmetric l.r. in $\mathcal{H}$. Then the set of all its nonnegative selfadjoint extensions consists of maximal and minimal elements. The maximal nonnegative selfadjoint extension is the Friedrichs extension $S_{\mathrm{F}}$ [46], which is associated with the closure of the sesquilinear form

$$
\mathfrak{s}[f, g]=\left(f^{\prime}, g\right),\left\{f, f^{\prime}\right\},\left\{g, g^{\prime}\right\} \in S .
$$

The minimal nonnegative selfadjoint extension $S_{\mathrm{K}}$ is called the Kreı̆n or the Krĕn-von Neumann extension and can be defined as follows [18]:

$$
S_{\mathrm{K}}=\left(\left(S^{-1}\right)_{\mathrm{F}}\right)^{-1} \text {. }
$$

The closed form $S_{\mathrm{F}}[\cdot, \cdot]$ is a closed restriction of the closed form $\widetilde{S}[\cdot, \cdot]$ associated with any nonnegative selfadjoint extension $\widetilde{S}$ of $S$ [34, 11]. A nonnegative selfadjoint extension $\widetilde{S}$ of $S$ is called extremal [1] if

$$
\inf \left\{\left(f^{\prime}-g^{\prime}, f-g\right):\left\{g, g^{\prime}\right\} \in S\right\}=0 \quad \text { for all } \quad\left\{f, f^{\prime}\right\} \in \widetilde{S} .
$$

It is proved in [11, Proposition 3] that if $\widetilde{S}$ is an extremal extension of $S$, then the closed form $\widetilde{S}[\cdot, \cdot]$ is a closed restriction of the closed form $S_{\mathrm{K}}[\cdot, \cdot]$ associated with the Krĕn extension of $S$.

Let $Q=\mathfrak{C}(S)$ be the Cayley transform of $S$. Then $Q$ is a non-densely defined Hermitian contraction with $\operatorname{dom} Q=\operatorname{ran}(S+I)$. There is a one-to-one correspondence [34, 18]

$$
\widetilde{Q}=\mathfrak{C}(\widetilde{S}), \widetilde{S}=\mathfrak{C}(\widetilde{Q})
$$

between the set of all nonnegative selfadjoint extensions and the set of all selfadjoint contractive extensions of $Q$. Let

$$
Q_{\mu}=\mathfrak{C}\left(S_{\mathrm{F}}\right), Q_{M}=\mathfrak{C}\left(S_{\mathrm{K}}\right) .
$$

The operators $Q_{\mu}$ and $Q_{M}$ posses the following properties [34]:

if $\mathfrak{N}:=\mathcal{H} \ominus \operatorname{dom} Q$, then

$$
\left(I+Q_{\mu}\right)_{\mathfrak{N}}=\left(I-Q_{M}\right)_{\mathfrak{N}}=0 .
$$

$\mathfrak{N}$ is the deficiency subspace of $S$ corresponding to $\lambda=-1$. Set $\mathfrak{N}_{0}:=\overline{\operatorname{ran}}\left(Q_{M}-Q_{\mu}\right)$. The set of all selfadjoint contractive extensions of $Q$ forms the operator interval $\left[Q_{\mu}, Q_{M}\right]$ [34], which admits the parameterizations

$$
\begin{aligned}
& (7.2) \quad \widetilde{Q}=\frac{1}{2}\left(Q_{\mu}+Q_{M}\right)+\frac{1}{2}\left(Q_{M}-Q_{\mu}\right)^{\frac{1}{2}} \widetilde{Z}\left(Q_{M}-Q_{\mu}\right)^{\frac{1}{2}} \\
& =Q_{\mu}+\frac{1}{2}\left(Q_{M}-Q_{\mu}\right)^{\frac{1}{2}}\left(I_{\mathfrak{N}_{0}}+\widetilde{Z}\right)\left(Q_{M}-Q_{\mu}\right)^{\frac{1}{2}}=Q_{M}-\frac{1}{2}\left(Q_{M}-Q_{\mu}\right)^{\frac{1}{2}}\left(I_{\mathfrak{N}_{0}}-\widetilde{Z}\right)\left(Q_{M}-Q_{\mu}\right)^{\frac{1}{2}} .
\end{aligned}
$$

A nonnegative selfadjoint extension $\widetilde{S}$ is extremal if and only if the parameter $\widetilde{Z}$ for $\widetilde{Q}=\mathfrak{C}(\widetilde{S})$ in the right hand side in (7.2) is a selfadjoint and unitary operator (a fundamental symmetry) in the subspace $\mathfrak{N}_{0}$ [12]. This is equivalent to the equality $\left(I-\widetilde{Q}^{2}\right)_{\mathfrak{N}}=0$, see [12]. If $\operatorname{dim} \mathfrak{N}_{0}=1$, then extremal extensions of $S$ are only $S_{\mathrm{F}}$ and $S_{\mathrm{K}}$.

From (17.2) and (17.1) it follows the equalities

$$
(I \pm \widetilde{Q})_{\mathfrak{N}}=\frac{1}{2}\left(Q_{M}-Q_{\mu}\right)^{\frac{1}{2}}\left(I_{\mathfrak{N}_{0}} \pm \widetilde{Z}\right)\left(Q_{M}-Q_{\mu}\right)^{\frac{1}{2}}
$$


Note that from (17.1) and (3.3), (3.8) one gets the equalities for shorted operators

$$
\left(S_{\mathrm{K}}\right)_{\mathfrak{N}}=0,\left(\left(S_{\mathrm{F}}\right)^{-1}\right)_{\mathfrak{N}}=0 .
$$

Moreover, the Kre ̌n uniqueness criteria [34] is equivalent to the equality $\left(S_{\mathrm{F}}\right)_{\mathfrak{N}}=0$.

The next theorem shows connections of the arithmetic and harmonic means with the theory of nonnegative selfadjoint extensions of nonnegative symmetric l.r..

Theorem 7.1. Let $S$ be a nonnegative symmetric l.r.. Then

(1) for an arbitrary nonnegative selfadjoint extensions $\widetilde{S}_{1}$ and $\widetilde{S}_{2}$ of $S$ their arithmetic mean $\frac{1}{2}\left(\widetilde{S}_{1}+S_{2}\right)$, harmonic mean $h\left(\widetilde{S}_{1}, \widetilde{S}_{2}\right)$, and nonnegative selfadjoint l.r. $c_{0}\left(\widetilde{S}_{1}, \widetilde{S}_{2}\right)$, given by (5.7), are nonnegative selfadjoint extensions of $S$;

(2) if $\widetilde{Z}_{1}$ and $\widetilde{Z}_{2}$ are parameters of $\mathfrak{C}\left(\widetilde{S}_{1}\right)$ and $\mathfrak{C}\left(\widetilde{S}_{2}\right)$ in formulae $(\overline{7.2})$, respectively, then the corresponding parameters for $\mathfrak{C}\left(\frac{1}{2}\left(\widetilde{S}_{1} \dot{+} S_{2}\right)\right), \mathfrak{C}\left(h\left(\widetilde{S}_{1}, S_{2}\right)\right)$ and $\mathfrak{C}\left(c_{0}\left(\widetilde{S}_{1}, \widetilde{S}_{2}\right)\right)$ are the operators

$h\left(I_{\mathfrak{N}_{0}}+\widetilde{Z}_{1}, I_{\mathfrak{N}_{0}}+\widetilde{Z}_{2}\right)-I_{\mathfrak{N}_{0}}, I_{\mathfrak{N}_{0}}-h\left(I_{\mathfrak{N}_{0}}-\widetilde{Z}_{1}, I_{\mathfrak{N}_{0}}-\widetilde{Z}_{2}\right), \quad$ and $\quad \frac{1}{2}\left(\widetilde{Z}_{1}+\widetilde{Z}_{2}\right)$,

respectively;

(3) for an arbitrary nonnegative selfadjoint extension $\widetilde{S}$ of $S$ the equalities

$$
\frac{1}{2}\left(S_{\mathrm{F}} \dot{+} \widetilde{S}\right)=S_{\mathrm{F}}, h\left(S_{\mathrm{K}}, \widetilde{S}\right)=S_{\mathrm{K}}
$$

hold;

(4) if $\widetilde{S}_{1}$ and $\widetilde{S}_{2}$ are extremal extensions of $S$, then $\frac{1}{2}\left(\widetilde{S}_{1} \dot{+} S_{2}\right)$ and $h\left(\widetilde{S}_{1}, \widetilde{S}_{2}\right)$ are extremal extensions of $S$ as well, moreover, for an arbitrary extremal extension $\widetilde{S}$ one has

$$
\frac{1}{2}\left(S_{\mathrm{K}} \dot{+} \widetilde{S}\right)=\widetilde{S}, h\left(S_{\mathrm{F}}, \widetilde{S}\right)=\widetilde{S} .
$$

Proof. If $\widetilde{S}$ is a nonnegative selfadjoint extension of $S$, then (see [11, 18, 29, 30])

- $S_{\mathrm{K}} \leq \widetilde{S} \leq S_{\mathrm{F}}$, the form $S_{\mathrm{F}}[\cdot, \cdot]$ is the closed restriction of the form $\widetilde{S}[\cdot, \cdot]$, and $\mathcal{D}[\widetilde{S}] \cap \mathcal{D}\left[S_{\mathrm{F}}\right]=\mathcal{D}\left[S_{\mathrm{F}}\right]$

- $\widetilde{S}^{-1}$ is a nonnegative selfadjoint extension of $S^{-1}, S_{\mathrm{F}}^{-1} \leq \widetilde{S}^{-1} \leq S_{\mathrm{K}}^{-1}$, the form $S_{\mathrm{K}}^{-1}[\cdot, \cdot]$ is the closed restriction of the form $\widetilde{S}^{-1}[\cdot, \cdot]$, and $\mathcal{D}\left[\widetilde{S}^{-1}\right] \cap \mathcal{D}\left[S_{\mathrm{K}}^{-1}\right]=\mathcal{D}\left[S_{\mathrm{K}}^{-1}\right]$.

Let $\widetilde{S}_{1}$ and $\widetilde{S}_{2}$ be two nonnegative selfadjoint extensions of $S$. Then

$$
\mathcal{D}\left[\widetilde{S}_{1}\right] \cap \mathcal{D}\left[\widetilde{S}_{2}\right] \supseteq \mathcal{D}\left[S_{\mathrm{F}}\right], \mathcal{D}\left[\widetilde{S}_{1}^{-1}\right] \cap \mathcal{D}\left[\widetilde{S}_{2}^{-1}\right] \supseteq \mathcal{D}\left[S_{\mathrm{K}}^{-1}\right],
$$

and

$$
\begin{aligned}
& \frac{1}{2}\left(\widetilde{S}_{1} \dot{+} \widetilde{S}_{2}\right)[f]=\frac{1}{2}\left(\widetilde{S}_{1}[f]+\widetilde{S}_{2}[f]\right) \geq S_{\mathrm{K}}[f], f \in \mathcal{D}\left[\widetilde{S}_{1}\right] \cap \mathcal{D}\left[\widetilde{S}_{2}\right], \\
& \frac{1}{2}\left(\widetilde{S}_{1} \dot{+} \widetilde{S}_{2}\right)[\varphi]=S_{\mathrm{F}}[\varphi], \varphi \in \mathcal{D}\left[S_{\mathrm{F}}\right], \\
& \frac{1}{2}\left(\widetilde{S}_{1}^{-1} \dot{+} \widetilde{S}_{2}^{-1}\right)[g]=\frac{1}{2}\left(\widetilde{S}_{1}^{-1}[g]+\widetilde{S}_{2}^{-1}[g]\right) \geq S_{\mathrm{F}}^{-1}[g], g \in \mathcal{D}\left[\widetilde{S}_{1}^{-1}\right] \cap \mathcal{D}\left[\widetilde{S}_{2}^{-1}\right], \\
& \frac{1}{2}\left(\widetilde{S}_{1}^{-1}+\widetilde{S}_{2}^{-1}\right)[\psi]=S_{\mathrm{K}}^{-1}[\psi], \psi \in \mathcal{D}\left[S_{\mathrm{K}}^{-1}\right] .
\end{aligned}
$$

Hence $\frac{1}{2}\left(\widetilde{S}_{1} \dot{+} \widetilde{S}_{2}\right)$ is a nonnegative selfadjoint extension of $S$ and $\frac{1}{2}\left(\widetilde{S}_{1}^{-1} \dot{+} \widetilde{S}_{2}^{-1}\right)$ is a nonnegative selfadjoint extension of $S^{-1}$. It follows that

$$
h\left(\widetilde{S}_{1}, \widetilde{S}_{2}\right)=2\left(\widetilde{S}_{1}^{-1} \dot{+} \widetilde{S}_{2}^{-1}\right)^{-1}
$$


is a nonnegative selfadjoint extension of $S$. Besides, we get equalities in (7.4).

Set $Q=\mathfrak{C}(S), Q_{\mu}=\mathfrak{C}\left(S_{\mathrm{F}}\right), Q_{M}=\mathfrak{C}\left(S_{\mathrm{K}}\right), \mathfrak{N}=\mathcal{H} \ominus \operatorname{dom} Q, \mathfrak{N}_{0}=\overline{\operatorname{ran}}\left(Q_{M}-Q_{\mu}\right)$.

Let $\widetilde{Q}_{1}:=\mathfrak{C}\left(\widetilde{S}_{1}\right)$ and $\widetilde{Q}_{2}:=\mathfrak{C}\left(\widetilde{S}_{2}\right)$. The operators $\widetilde{Q}_{k}, k=1,2$ admit the representations by means of expression (7.2):

$$
\widetilde{Q}_{k}=\frac{1}{2}\left(Q_{\mu}+Q_{M}\right)+\frac{1}{2}\left(Q_{M}-Q_{\mu}\right)^{\frac{1}{2}} \widetilde{Z}_{k}\left(Q_{M}-Q_{\mu}\right)^{\frac{1}{2}},
$$

where $\widetilde{Z}_{k}, k=1,2$ are selfadjoint contractions in $\mathfrak{N}_{0}$. Since the operator $\frac{1}{2}\left(\widetilde{Z}_{1}+\widetilde{Z}_{2}\right)$ is a selfadjoint contraction and

$$
\frac{1}{2}\left(\widetilde{Q}_{1}+\widetilde{Q}_{2}\right)=\frac{1}{2}\left(Q_{\mu}+Q_{M}\right)+\frac{1}{2}\left(Q_{M}-Q_{\mu}\right)^{\frac{1}{2}}\left(\frac{1}{2}\left(\widetilde{Z}_{1}+\widetilde{Z}_{2}\right)\right)\left(Q_{M}-Q_{\mu}\right)^{\frac{1}{2}},
$$

the operator $\frac{1}{2}\left(\widetilde{Q}_{1}+\widetilde{Q}_{2}\right)$ is a selfadjoint contractive extension of $Q$. Hence $c_{0}\left(\widetilde{S}_{1}, \widetilde{S}_{2}\right)=$ $\mathfrak{C}\left(\frac{1}{2}\left(\widetilde{Q}_{1}+\widetilde{Q}_{2}\right)\right)$ is a nonnegative selfadjoint extension of $S$.

From (5.2) and (5.3) we get the equalities

$$
I+\mathfrak{C}\left(\frac{1}{2}\left(\widetilde{S}_{1} \dot{+} \widetilde{S}_{2}\right)\right)=h\left(I+\widetilde{Q}_{1}, I+\widetilde{Q}_{2}\right), I-\mathfrak{C}\left(h\left(\widetilde{S}_{1}, \widetilde{S}_{2}\right)\right)=h\left(I-\widetilde{Q}_{1}, I-\widetilde{Q}_{2}\right) .
$$

Further, using (1.11), one obtains

$$
\left(I+\mathfrak{C}\left(\frac{1}{2}\left(\widetilde{S}_{1} \dot{+} \widetilde{S}_{2}\right)\right)\right)_{\mathfrak{N}}=\left(h\left(I+\widetilde{Q}_{1}, I+\widetilde{Q}_{2}\right)\right)_{\mathfrak{N}}=h\left(\left(I+\widetilde{Q}_{1}\right)_{\mathfrak{N}},\left(I+\widetilde{Q}_{2}\right)_{\mathfrak{N}}\right) .
$$

From (7.3) and [10, Proposition 1] it follows the equality

$$
\begin{aligned}
&\left(\left(I+\widetilde{Q}_{1}\right)_{\mathfrak{N}},\left(I+\widetilde{Q}_{2}\right)_{\mathfrak{N}}\right) \\
&=2\left(\frac{1}{2}\left(Q_{M}-Q_{\mu}\right)^{\frac{1}{2}}\left(I_{\mathfrak{N}_{0}}+\widetilde{Z}_{1}\right)\left(Q_{M}-Q_{\mu}\right)^{\frac{1}{2}}\right):\left(\frac{1}{2}\left(Q_{M}-Q_{\mu}\right)^{\frac{1}{2}}\left(I_{\mathfrak{N}_{0}}+\widetilde{Z}_{2}\right)\left(Q_{M}-Q_{\mu}\right)^{\frac{1}{2}}\right) \\
&=\frac{1}{2}\left(Q_{M}-Q_{\mu}\right)^{\frac{1}{2}} h\left(I_{\mathfrak{N}_{0}}+\widetilde{Z}_{1}, I_{\mathfrak{N}_{0}}+\widetilde{Z}_{2}\right)\left(Q_{M}-Q_{\mu}\right)^{\frac{1}{2}} .
\end{aligned}
$$

Now from (7.2), (17.3) and the uniqueness of the representation we get that

$$
\mathfrak{C}\left(\frac{1}{2}\left(\widetilde{S}_{1}+\widetilde{S}_{2}\right)\right)=\frac{1}{2}\left(Q_{\mu}+Q_{M}\right)+\frac{1}{2}\left(Q_{M}-Q_{\mu}\right)^{\frac{1}{2}} \widetilde{Z}\left(Q_{M}-Q_{\mu}\right)^{\frac{1}{2}}
$$

where $\widetilde{Z}=h\left(I_{\mathfrak{N}_{0}}+\widetilde{Z}_{1}, I_{\mathfrak{N}_{0}}+\widetilde{Z}_{2}\right)-I_{\mathfrak{N}_{0}}$. Similarly

$$
\mathfrak{C}\left(h\left(\widetilde{S}_{1}, \widetilde{S}_{2}\right)\right)=\frac{1}{2}\left(Q_{\mu}+Q_{M}\right)+\frac{1}{2}\left(Q_{M}-Q_{\mu}\right)^{\frac{1}{2}} \widetilde{W}\left(Q_{M}-Q_{\mu}\right)^{\frac{1}{2}},
$$

where $\widetilde{W}=I_{\mathfrak{N}_{0}}-h\left(I_{\mathfrak{N}_{0}}-\widetilde{Z}_{1}, I_{\mathfrak{N}_{0}}-\widetilde{Z}_{2}\right)$.

Let $\widetilde{S}$ be an extremal nonnegative selfadjoint extension of $S$ and let $\widetilde{Q}=\mathfrak{C}(\widetilde{S})$ be its Cayley transform. Then $\widetilde{Q}$ admits the representation (7.2) with a fundamental symmetry $\widetilde{Z}$ in $\mathfrak{N}_{0}$. The operator $\widetilde{S}^{-1}$ is a nonnegative selfadjoint extension of the operator $S^{-1}$ and

$$
\mathfrak{C}\left(S^{-1}\right)=-Q, \mathfrak{C}\left(\left(S^{-1}\right)_{\mathrm{F}}\right)=-Q_{M}, \mathfrak{C}\left(\left(S^{-1}\right)_{\mathrm{K}}\right)=-Q_{\mu}, \mathfrak{C}\left(\widetilde{S}^{-1}\right)=-\widetilde{Q}
$$

Hence

$$
-\widetilde{Q}=-\frac{1}{2}\left(Q_{\mu}+Q_{M}\right)+\frac{1}{2}\left(Q_{M}-Q_{\mu}\right)^{\frac{1}{2}}(-\widetilde{Z})\left(Q_{M}-Q_{\mu}\right)^{\frac{1}{2}}
$$


Because $\widetilde{Z}$ is a selfadjoint and unitary (in $\mathfrak{N}_{0}$ ), the operator $-\widetilde{Z}$ is selfadjoint and unitary as well. Therefore, the operator $\widetilde{S}^{-1}$ is an extremal extension of $S^{-1}$.

Let $\widetilde{S}_{1}$ and $\widetilde{S}_{2}$ be two extremal nonnegative selfadjoint extensions of $S$. Then the closed form $\widetilde{S}_{1}[\cdot, \cdot]$ and $\widetilde{S}_{2}[\cdot, \cdot]$ are closed restrictions of the closed form $S_{\mathrm{K}}[\cdot, \cdot]$. Therefore the sesquilinear form $\frac{1}{2}\left(\widetilde{S}_{1}+\widetilde{S}_{2}\right)[\cdot, \cdot]$ is a closed restriction of the closed form $S_{\mathrm{K}}[\cdot, \cdot]$. It follows that $\frac{1}{2}\left(\widetilde{S}_{1} \dot{+} \widetilde{S}_{2}\right)$ is an extremal extension of $S$.

Since $\widetilde{S}_{1}^{-1}$ and $\widetilde{S}_{2}^{-1}$ are extremal nonnegative selfadjoint extensions of $S^{-1}, \frac{1}{2}\left(\widetilde{S}_{1}^{-1} \dot{+} \widetilde{S}_{2}^{-1}\right)$ is an extremal extension of $S^{-1}$ and this implies that

$$
h\left(\widetilde{S}_{1}, \widetilde{S}_{2}\right)=\left(\frac{1}{2}\left(\widetilde{S}_{1}^{-1} \dot{+} \widetilde{S}_{2}^{-1}\right)\right)^{-1}
$$

is an extremal extension of $S$.

For an arbitrary extremal extension $\widetilde{S}$ of $S$ we have equalities

$$
\frac{1}{2}\left(S_{\mathrm{K}} \dot{+} \widetilde{S}\right)[f]=\widetilde{S}[f] \quad \forall f \in \mathcal{D}\left[\left(S_{\mathrm{K}} \dot{+} \widetilde{S}\right) / 2\right]=\mathcal{D}[\widetilde{S}]
$$

and

$$
\frac{1}{2}\left(S_{\mathrm{F}}^{-1} \dot{+} \widetilde{S}^{-1}\right)[h]=\widetilde{S}^{-1}[h] \quad \forall h \in \mathcal{D}\left[\left(S_{\mathrm{F}}^{-1} \dot{+} \widetilde{S}^{-1}\right) / 2\right]=\mathcal{D}\left[\widetilde{S}^{-1}\right] .
$$

It follows that equalities in (7.5) are valid.

The proof is complete.

Proposition 7.2. Let $S$ be a nonnegative symmetric l.r. in $\mathcal{H}$, having a non-unique nonnegative selfadjoint extension. Then the arithmetic-harmonic means ah $\left(\widetilde{S}_{1}, \widetilde{S}_{2}\right)$ of an arbitrary two extremal nonnegative selfadjoint extensions $\widetilde{S}_{1}, \widetilde{S}_{2}$ are extremal nonnegative selfadjoint extensions too. Moreover,

$$
\operatorname{ah}\left(\widetilde{S}_{1}, \widetilde{S}_{2}\right)=\left\langle\widetilde{S}_{1}^{(1)}, \widetilde{S}_{2}^{(1)}\right\rangle
$$

where $\widetilde{S}_{1}^{(1)}=\frac{1}{2}\left(\widetilde{S}_{1} \dot{+} \widetilde{S}_{2}\right), \widetilde{S}_{2}^{(1)}=h\left(\widetilde{S}_{1}, \widetilde{S}_{2}\right)$. In particular, ah $\left(S_{\mathrm{F}}, S_{\mathrm{K}}\right)=\left\langle S_{\mathrm{F}}, S_{\mathrm{K}}\right\rangle$.

Proof. Set $A=S_{\mathrm{F}}, B=S_{\mathrm{K}}$. From Theorem 7.1 it follows that

$$
h(A, B)=h\left(S_{\mathrm{F}}, S_{\mathrm{K}}\right)=S_{\mathrm{K}}=B, \frac{1}{2}(A \dot{+} B)=\frac{1}{2}\left(S_{\mathrm{F}} \dot{+} S_{\mathrm{K}}\right)=S_{\mathrm{F}}=A .
$$

Then from (6.1) and (6.2) we get $A_{\infty}=S_{\mathrm{F}}$ and $B_{\infty}=S_{\mathrm{K}}$.

Recall [26, Theorem 4.3] that if $\mathcal{L}_{1}, \mathcal{L}_{2}$ are two subspaces in the Hilbert space $\mathcal{H}$, then

$$
2\left(P_{\mathcal{L}_{1}}: P_{\mathcal{L}_{2}}\right)=P_{\mathcal{L}_{1} \cap \mathcal{L}_{2}} .
$$

Let $\widetilde{S}_{k}, k=1,2$ be two extremal extensions of $S$. Then the corresponding operators $\widetilde{Z}_{k}$, $k=1,2$ in (17.2) for $\mathfrak{C}\left(\widetilde{S}_{k}\right)$ are fundamental symmetries in $\mathfrak{N}_{0}$. Therefore, the operators $\frac{1}{2}\left(I_{\mathfrak{N}_{0}} \pm \widetilde{Z}_{k}\right)$ are orthogonal projections in $\mathfrak{N}_{0}$. Hence, the operators

$$
2\left(\frac{1}{2}\left(I_{\mathfrak{N}_{0}} \pm \widetilde{Z}_{1}\right)\right):\left(\frac{1}{2}\left(I_{\mathfrak{N}_{0}} \pm \widetilde{Z}_{2}\right)\right)=\frac{1}{2} h\left(I_{\mathfrak{N}_{0}} \pm \widetilde{Z}_{1}, I_{\mathfrak{N}_{0}} \pm \widetilde{Z}_{2}\right)
$$

are orthogonal projections in $\mathfrak{N}_{0}$. It follows that the operators

$$
\widetilde{Z}_{1}^{(1)}=h\left(I_{\mathfrak{N}_{0}}+\widetilde{Z}_{1}, I_{\mathfrak{N}_{0}}+\widetilde{Z}_{2}\right)-I_{\mathfrak{N}_{0}}, \widetilde{Z}_{2}^{(1)}=I_{\mathfrak{N}_{0}}-h\left(I_{\mathfrak{N}_{0}}-\widetilde{Z}_{1}, I_{\mathfrak{N}_{0}}-\widetilde{Z}_{2}\right)
$$


are fundamental symmetries in $\mathfrak{N}_{0}$. Define two sequences

$$
\widetilde{S}_{1}^{(0)}:=\widetilde{S}_{1}, \widetilde{S}_{2}^{(0)}:=\widetilde{S}_{2}, \widetilde{S}_{1}^{(n)}=\frac{1}{2}\left(\widetilde{S}_{1}^{(n-1)} \dot{+} \widetilde{S}_{2}^{(n-1)}\right), \widetilde{S}_{2}^{(n)}=h\left(\widetilde{S}_{1}^{(n-1)}, \widetilde{S}_{2}^{(n-1)}\right), n \in \mathbb{N} .
$$

Let us show that

$$
\widetilde{S}_{1}^{(n)}=\widetilde{S}_{1}^{(1)}, \widetilde{S}_{2}^{(n)}=\widetilde{S}_{2}^{(1)} n \geq 2 .
$$

Define also two sequences of operators in $\mathfrak{N}_{0}$ :

$$
\begin{aligned}
& \widetilde{Z}_{1}^{(n)}=h\left(I_{\mathfrak{N}_{0}}+\widetilde{Z}_{1}^{(n-1)}, I_{\mathfrak{N}_{0}}+\widetilde{Z}_{2}^{(n-1)}\right)-I_{\mathfrak{N}_{0}}, \\
& \widetilde{Z}_{2}^{(n)}=I_{\mathfrak{N}_{0}}-h\left(I_{\mathfrak{N}_{0}}-\widetilde{Z}_{1}^{(n-1)}, I_{\mathfrak{N}_{0}}-\widetilde{Z}_{2}^{(n-1)}\right), n \geq 2 .
\end{aligned}
$$

By Theorem 7.1 the operators $\widetilde{Z}_{1}^{(n)}$ and $\widetilde{Z}_{2}^{(n)}$ correspond for all $n \in \mathbb{N}$ to $\widetilde{Q}_{1}^{(n)}=\mathfrak{C}\left(\widetilde{S}_{1}^{(n)}\right)$, $\widetilde{Q}_{2}^{(n)}=\mathfrak{C}\left(\widetilde{S}_{2}^{(n)}\right)$ in their representation in $(7.2)$, respectively.

Because $\widetilde{S}_{2}^{(1)} \leq \widetilde{S}_{1}^{(1)}$, we get $\widetilde{Z}_{2}^{(1)} \geq \widetilde{Z}_{1}^{(1)}$. Hence

$$
I_{\mathfrak{N}_{0}}+\widetilde{Z}_{2}^{(1)} \geq I_{\mathfrak{N}_{0}}+\widetilde{Z}_{1}^{(1)}, I_{\mathfrak{N}_{0}}-\widetilde{Z}_{1}^{(1)} \geq I_{\mathfrak{N}_{0}}-\widetilde{Z}_{2}^{(1)} .
$$

Since the operators $\frac{1}{2}\left(I_{\mathfrak{N}_{0}} \pm \widetilde{Z}_{1}^{(1)}\right), \frac{1}{2}\left(I_{\mathfrak{N}_{0}} \pm \widetilde{Z}_{2}^{(1)}\right)$ are orthogonal projections in $\mathfrak{N}_{0}$, the equalities

$$
I_{\mathfrak{N}_{0}}+\widetilde{Z}_{1}^{(2)}=h\left(I_{\mathfrak{N}_{0}}+\widetilde{Z}_{1}^{(1)}, I_{\mathfrak{N}_{0}}+\widetilde{Z}_{2}^{(1)}\right), I_{\mathfrak{N}_{0}}-\widetilde{Z}_{2}^{(2)}=h\left(I_{\mathfrak{N}_{0}}-\widetilde{Z}_{1}^{(1)}, I_{\mathfrak{N}_{0}}-\widetilde{Z}_{2}^{(1)}\right)
$$

and equality (7.6) imply that

$$
I_{\mathfrak{N}_{0}}+\widetilde{Z}_{1}^{(2)}=I_{\mathfrak{N}_{0}}+\widetilde{Z}_{1}^{(1)}, I_{\mathfrak{N}_{0}}-\widetilde{Z}_{2}^{(2)}=I_{\mathfrak{N}_{0}}-\widetilde{Z}_{2}^{(1)} .
$$

Therefore $\widetilde{Z}_{1}^{(2)}=\widetilde{Z}_{1}^{(1)}, \widetilde{Z}_{2}^{(2)}=\widetilde{Z}_{2}^{(1)}$, and by induction

$$
\widetilde{Z}_{1}^{(n)}=\widetilde{Z}_{1}^{(1)}, \widetilde{Z}_{2}^{(n)}=\widetilde{Z}_{2}^{(1)}, \widetilde{S}_{1}^{(n)}=\widetilde{S}_{1}^{(1)}, \widetilde{S}_{2}^{(n)}=\widetilde{S}_{2}^{(1)} \forall n \geq 2 .
$$

Thus, $a h\left(\widetilde{S}_{1}, \widetilde{S}_{2}\right)=\left\langle\widetilde{S}_{1}^{(1)}, \widetilde{S}_{2}^{(1)}\right\rangle$. By Theorem 7.1 the operators $\widetilde{S}_{1}^{(1)}, \widetilde{S}_{2}^{(1)}$ are extremal nonnegative selfadjoint extensions of $S$.

Proposition 7.3. Consider in the Hilbert space $\mathcal{L}_{2}\left(\mathbb{R}_{+}\right)$the following differential operators

$$
L_{c} f=-\frac{d^{2} f}{d x^{2}},\left\{\begin{array}{l}
\operatorname{dom} L_{c}=\left\{f \in W_{2}^{2}\left(\mathbb{R}_{+}\right), f^{\prime}(0)=c f(0)\right\}, c>0, c \neq \infty, \\
\operatorname{dom} L_{0}=\left\{f \in W_{2}^{2}\left(\mathbb{R}_{+}\right), f^{\prime}(0)=0\right\} \\
\operatorname{dom} L_{\infty}=\left\{f \in W_{2}^{2}\left(\mathbb{R}_{+}\right), f(0)=0\right\}
\end{array}\right.
$$

where $W_{2}^{2}\left(\mathbb{R}_{+}\right)$is the Sobolev space. Then

$$
\begin{aligned}
& \frac{1}{2}\left(L_{c} \dot{+} L_{d}\right)=L_{\frac{1}{2}(c+d)}, c_{0}\left(L_{c}, L_{d}\right)=L_{c_{0}(c, d)}, h\left(L_{c}, L_{d}\right)=L_{h(c, d)}=L_{\frac{2 c d}{c+d}}, a h\left(L_{c}, L_{d}\right)=L_{\sqrt{c d}}, \\
& \frac{1}{2}\left(L_{c} \dot{+} L_{\infty}\right)=L_{\infty}, \quad c_{0}\left(L_{c}, L_{\infty}\right)=L_{2 c+1}, h\left(L_{c}, L_{\infty}\right)=L_{2 c}, a h\left(L_{c}, L_{\infty}\right)=L_{\infty}, c>0 \\
& \frac{1}{2}\left(L_{c} \dot{+} L_{0}\right)=L_{c / 2}, \quad c_{0}\left(L_{c}, L_{0}\right)=L_{\frac{c}{2+c}}, h\left(L_{c}, L_{0}\right)=L_{0}, a h\left(L_{c}, L_{0}\right)=L_{0}, c>0 \\
& \frac{1}{2}\left(L_{0} \dot{+} L_{\infty}\right)=L_{\infty}, c_{0}\left(L_{0}, L_{\infty}\right)=L_{1}, h\left(L_{0}, L_{\infty}\right)=L_{0}, a h\left(L_{\infty}, L_{0}\right)=\left\langle L_{\infty}, L_{0}\right\rangle .
\end{aligned}
$$

Proof. The operators $L_{c}$ are nonnegative selfadjoint extensions of the nonnegative symmetric operator

$$
S f=-\frac{d^{2} f}{d x^{2}}, \operatorname{dom} S=\left\{f \in W_{2}^{2}\left(\mathbb{R}_{+}\right), f(0)=f^{\prime}(0)=0\right\} .
$$

The operator $S$ has one-dimensional deficiency subspaces. In particular,

$$
\mathfrak{N}=\{\xi \exp (-x), \xi \in \mathbb{C}\}
$$


is the deficiency subspace corresponding to $\lambda=-1$.

One can show that

$$
\left(L_{c}+I\right)^{-1} \exp (-x)=\frac{1}{2} \exp (-x)\left(x+\frac{1}{c+1}\right), x \in \mathbb{R}_{+} .
$$

Then

$$
\begin{aligned}
& \left(\left(L_{c}+I\right)^{-1}-\left(L_{\infty}+I\right)^{-1}\right) \exp (-x)=\frac{\exp (-x)}{2(c+1)}, \\
& \left(\left(L_{0}+I\right)^{-1}-\left(L_{\infty}+I\right)^{-1}\right) \exp (-x)=\frac{1}{2} \exp (-x) .
\end{aligned}
$$

The operators $L_{\infty}$ and $L_{0}$ are the Friedrichs and the Kreln extension of $S$, respectively. Hence as it is shown above

$$
\frac{1}{2}\left(L_{0} \dot{+} L_{\infty}\right)=L_{\infty}, h\left(L_{0}, L_{\infty}\right)=L_{0}, a h\left(L_{\infty}, L_{0}\right)=\left\langle L_{\infty}, L_{0}\right\rangle .
$$

Set

From (1.12) we have

$$
\widetilde{Q}_{c}=\mathfrak{C}\left(L_{c}\right), Q_{\mu}=\mathfrak{C}\left(L_{\infty}\right), Q_{M}=\mathfrak{C}\left(L_{0}\right)
$$

$$
\widetilde{Q}_{c}-Q_{\mu}=2\left(\left(L_{c}+I\right)^{-1}-\left(L_{\infty}+I\right)^{-1}\right), Q_{M}-Q_{\mu}=2\left(\left(L_{0}+I\right)^{-1}-\left(L_{\infty}+I\right)^{-1}\right)
$$

and $\operatorname{ran}\left(\widetilde{Q}_{c}-Q_{\mu}\right)=\operatorname{ran}\left(Q_{M}-Q_{\mu}\right)=\mathfrak{N}$. Now from (7.2) it follows that

$$
2\left(\left(L_{c}+I\right)^{-1}-\left(L_{\infty}+I\right)^{-1}\right)=\left(1+\widetilde{z}_{c}\right)\left(\left(L_{0}+I\right)^{-1}-\left(L_{\infty}+I\right)^{-1}\right) .
$$

Using (7.7) we obtain

Hence

$$
\widetilde{z}_{c}=\frac{1-c}{1+c}, z_{0}=1, z_{\infty}=-1
$$

$$
h\left(1+\widetilde{z}_{d}, 1+\widetilde{z}_{d}\right)-1=\frac{1-\frac{1}{2}(c+d)}{1+\frac{1}{2}(c+d)}, 1-h\left(1-\widetilde{z}_{d}, 1-\widetilde{z}_{d}\right)=\frac{1-\frac{2 c d}{c+d}}{1+\frac{2 c d}{c+d}}
$$

Applying Theorem 7.1 and (5.7), (5.8), we get

$$
\begin{aligned}
& \frac{1}{2}\left(L_{c} \dot{+} L_{d}\right)=L_{\frac{1}{2}(c+d)}, c_{0}\left(L_{c}, L_{d}\right)=L_{c_{0}(c, d)}, h\left(L_{c}, L_{d}\right)=L_{h(c, d)}=L_{\frac{2 c d}{c+d}}, \\
& \frac{1}{2}\left(L_{c} \dot{+} L_{\infty}\right)=L_{\infty}, c_{0}\left(L_{c}, L_{\infty}\right)=L_{2 c+1}, h\left(L_{c}, L_{\infty}\right)=L_{2 c}, \\
& \frac{1}{2}\left(L_{c} \dot{+} L_{0}\right)=L_{c / 2}, c_{0}\left(L_{c} L_{0}\right)=L_{\frac{c}{2+c}}, h\left(L_{c}, L_{0}\right)=L_{0} .
\end{aligned}
$$

Set

$$
\begin{gathered}
A_{1}=\frac{1}{2}\left(L_{c} \dot{+} L_{d}\right), B_{1}=h\left(L_{c}, L_{d}\right), \ldots, A_{n}=\frac{1}{2}\left(A_{n-1} \dot{+} B_{n-1}\right), B_{n}=h\left(A_{n-1}, B_{n-1}\right), n \geq 2, \\
c_{1}=\frac{1}{2}(c+d), d_{1}=h(c, d)=\frac{2 c d}{c+d}, \ldots, \\
c_{n}=\frac{1}{2}\left(c_{n-1}+d_{n-1}\right), d_{n}=h\left(c_{n-1}, d_{n-1}\right)=\frac{2 c_{n-1} d_{n-1}}{c_{n-1}+d_{n-1}}, n \geq 2 . \\
\widetilde{z}_{c_{n}}=\frac{1-c_{n}}{1+c_{n}}, \widetilde{z}_{d_{n}}=\frac{1-d_{n}}{1+d_{n}}, n \in \mathbb{N} .
\end{gathered}
$$

Then

$$
A_{n}=L_{c_{n}}, B_{n}=L_{d_{n}} \forall n \in \mathbb{N} .
$$


Due to Theorem 7.1 we have the equalities for the Cayley transforms

$$
\begin{aligned}
& \mathfrak{C}\left(A_{n}\right)=\frac{1}{2}\left(Q_{\mu}+Q_{M}\right)+\frac{1}{2} \widetilde{z}_{c_{n}}\left(Q_{M}-Q_{\mu}\right), \\
& \mathfrak{C}\left(B_{n}\right)=\frac{1}{2}\left(Q_{\mu}+Q_{M}\right)+\frac{1}{2} \widetilde{z}_{d_{n}}\left(Q_{M}-Q_{\mu}\right), n \in \mathbb{N} .
\end{aligned}
$$

From

$$
\lim _{n \rightarrow \infty} c_{n}=\lim _{n \rightarrow \infty} d_{n}=\sqrt{c d}
$$

it follows that

$$
\mathrm{s}-\lim _{n \rightarrow \infty} \mathfrak{C}\left(A_{n}\right)=\mathrm{s}-\lim _{n \rightarrow \infty} \mathfrak{C}\left(B_{n}\right)=\frac{1}{2}\left(Q_{\mu}+Q_{M}\right)+\frac{1-\sqrt{c d}}{1+\sqrt{c d}} \frac{Q_{M}-Q_{\mu}}{2} .
$$

Therefore

$$
\mathrm{s}-\mathrm{R}-\lim _{n \rightarrow \infty} L_{c_{n}}=\mathrm{s}-\mathrm{R}-\lim _{n \rightarrow \infty} L_{d_{n}}=L_{\sqrt{c d}} .
$$

Thus, $a h\left(L_{c}, L_{d}\right)=L_{\sqrt{c d}}$ for the case $\{c>0, d \in[0,+\infty]\}$.

Observe that the equality

$$
\operatorname{dim} \operatorname{ran}\left(\left(S_{\mathrm{K}}+I\right)^{-1}-\left(S_{\mathrm{F}}+I\right)^{-1}\right)=\operatorname{dim}\left(\mathcal{D}\left[S_{\mathrm{K}}\right] / \mathcal{D}\left[S_{\mathrm{F}}\right]\right)
$$

takes place [34]. Besides if

$$
\operatorname{dim} \operatorname{ran}\left(\left(S_{\mathrm{K}}+I\right)^{-1}-\left(S_{\mathrm{F}}+I\right)^{-1}\right)=1,
$$

then for an arbitrary nonnegative selfadjoint extension $\widetilde{S}$ of $S$ from (1.12) and (7.2) it follows that

$$
(\widetilde{S}+I)^{-1}=\frac{1}{2}\left(\left(S_{\mathrm{K}}+I\right)^{-1}+\left(S_{\mathrm{F}}+I\right)^{-1}\right)+\frac{\widetilde{z}}{2}\left(\left(S_{\mathrm{K}}+I\right)^{-1}-\left(S_{\mathrm{F}}+I\right)^{-1}\right),
$$

for some $\widetilde{z} \in[-1,1]$.

Theorem 7.4. Let $S$ be a nonnegative symmetric l.r. such that (17.8) holds. Suppose that $\widetilde{S}_{1}$ and $\widetilde{S}_{2}$ are two different non-extremal nonnegative selfadjoint extensions of $S$. Then the arithmetic-harmonic mean ah $\left(\widetilde{S}_{1}, \widetilde{S}_{2}\right)$ is singleton. Moreover, if the numbers $\widetilde{z}_{1}, \widetilde{z}_{2} \in[-1,1]$ correspond to $\widetilde{S}_{1}$ and $\widetilde{S}_{2}$ in the resolvent formula (7.9), then

$$
\begin{aligned}
\left(a h\left(\widetilde{S}_{1}, \widetilde{S}_{2}\right)+I\right)^{-1}=\frac{1}{2}\left(\left(S_{\mathrm{K}}+I\right)^{-1}+\left(S_{\mathrm{F}}+I\right)^{-1}\right) & \\
& +\frac{\widetilde{w}}{2}\left(\left(S_{\mathrm{K}}+I\right)^{-1}-\left(S_{\mathrm{F}}+I\right)^{-1}\right),
\end{aligned}
$$

where

$$
\widetilde{w}=\frac{\sqrt{\left(1+\widetilde{z}_{1}\right)\left(1+\widetilde{z}_{2}\right)}-\sqrt{\left(1-\widetilde{z}_{1}\right)\left(1-\widetilde{z}_{2}\right)}}{\sqrt{\left(1+\widetilde{z}_{1}\right)\left(1+\widetilde{z}_{2}\right)}+\sqrt{\left(1-\widetilde{z}_{1}\right)\left(1-\widetilde{z}_{2}\right)}} .
$$

In particular, ah $\left(S_{\mathrm{F}}, \widetilde{S}\right)=S_{\mathrm{F}}$, when $\widetilde{S} \neq S_{\mathrm{K}}$ and ah $\left(S_{\mathrm{K}}, \widetilde{S}\right)=S_{\mathrm{K}}$, when $\widetilde{S} \neq S_{\mathrm{F}}$.

Proof. Clearly, $\left\langle\widetilde{S}_{1}, \widetilde{S}_{2}\right\rangle \neq\left\langle\widetilde{S}_{\mathrm{F}}, \widetilde{S}_{\mathrm{K}}\right\rangle$. Define the iterations

$$
\widetilde{S}_{1}^{(0)}:=\widetilde{S}_{1}, \widetilde{S}_{2}^{(0)}:=\widetilde{S}_{2}, \widetilde{S}_{1}^{(n)}=\frac{1}{2}\left(\widetilde{S}_{1}^{(n-1)}+\widetilde{S}_{2}^{(n-1)}\right), \widetilde{S}_{2}^{(n)}=h\left(\widetilde{S}_{1}^{(n-1)}, S_{2}^{(n-1)}\right), n \in \mathbb{N} .
$$


Then from (7.9) and Theorem 7.1

$\left(\widetilde{S}_{j}^{(n)}+I\right)^{-1}=\frac{1}{2}\left(\left(S_{\mathrm{K}}+I\right)^{-1}+\left(S_{\mathrm{F}}+I\right)^{-1}\right)+\frac{\widetilde{z}_{j}^{(n)}}{2}\left(\left(S_{\mathrm{K}}+I\right)^{-1}-\left(S_{\mathrm{F}}+I\right)^{-1}\right), j=1,2, n \in \mathbb{N}$, where $\widetilde{z}_{1}^{(0)}=\widetilde{z}_{1}, \widetilde{z}_{2}^{(0)}=\widetilde{z}_{2}$,

$$
\left\{\begin{array}{l}
1+\widetilde{z}_{1}^{(n)}=h\left(1+\widetilde{z}_{1}^{(n-1)}, 1+\widetilde{z}_{2}^{(n-1)}\right)=\frac{2\left(1+\widetilde{z}_{1}^{(n-1)}\right)\left(1+\widetilde{z}_{2}^{(n-1)}\right)}{2+\widetilde{z}_{1}^{(n-1)}+\widetilde{z}_{2}^{(n-1)}} \\
1-\widetilde{z}_{2}^{(n)}=h\left(1-\widetilde{z}_{1}^{(n-1)}, 1-\widetilde{z}_{2}^{(n-1)}\right)=\frac{2\left(1-\widetilde{z}_{1}^{(n-1)}\right)\left(1-\widetilde{z}_{2}^{(n-1)}\right)}{2-\widetilde{z}_{1}^{(n-1)}-\widetilde{z}_{2}^{(n-1)}}
\end{array}\right.
$$

Besides, $\left\{\widetilde{z}_{1}^{(n)}\right\}$ and $\left\{\widetilde{z}_{2}^{(n)}\right\}$ are non-decreasing and non-increasing sequences, respectively, and $\widetilde{z}_{1}^{(n)} \leq \widetilde{z}_{2}^{(n)} \forall n \in \mathbb{N}$. Hence the sequences $\left\{\widetilde{z}_{1}^{(n)}\right\}$ and $\left\{\widetilde{z}_{2}^{(n)}\right\}$ converge. Set

$$
\widetilde{w}_{j}=\lim _{n \rightarrow \infty} \widetilde{z}_{j}^{(n)}, j=1,2 .
$$

Note that because $\left\langle\widetilde{S}_{1}, \widetilde{S}_{2}\right\rangle \neq\left\langle\widetilde{S}_{\mathrm{F}}, \widetilde{S}_{\mathrm{K}}\right\rangle$ we have $\widetilde{z}_{1} \widetilde{z}_{2} \neq-1$.

If $\widetilde{z}_{1}=-1, \widetilde{z}_{2} \neq 1$, then from $(7.12)$ one has $\widetilde{z}_{1}^{(n)}=-1$ for all $n \in \mathbb{N}$ and therefore $\widetilde{w}_{1}=\widetilde{w}_{2}=-1$. If $\widetilde{z}_{2}=1, \widetilde{z}_{1} \neq-1$, then $\widetilde{w}_{1}=\widetilde{w}_{2}=1$.

Suppose $\widetilde{z}_{1} \neq-1, \widetilde{z}_{2} \neq-1$. Then (7.12) yields $\widetilde{w}_{1}=\widetilde{w}_{2}:=\widetilde{w}$. Let us find $\widetilde{w}$. Set

$$
a_{n}:=\mathfrak{C}\left(\widetilde{z}_{1}^{(n)}\right)=\frac{1-\widetilde{z}_{1}^{(n)}}{1+\widetilde{z}_{1}^{(n)}}, b_{n}:=\mathfrak{C}\left(\widetilde{z}_{2}^{(n)}\right)=\frac{1-\widetilde{z}_{2}^{(n)}}{1+\widetilde{z}_{n}^{(n)}}, n \in \mathbb{N}_{0} .
$$

Then, using (5.2) and (5.3), we obtain

$$
a_{n}=\frac{1}{2}\left(a_{n-1}+b_{n-1}\right), b_{n}=h\left(a_{n-1}, b_{n-1}\right)=\frac{2 a_{n-1} b_{n-1}}{a_{n-1}+b_{n-1}}, n \in \mathbb{N}_{0} .
$$

Hence,

$$
\lim _{n \rightarrow \infty} a_{n}=\lim _{n \rightarrow \infty} b_{n}=\sqrt{a_{0} b_{0}}=\sqrt{\frac{1-\widetilde{z}_{1}}{1+\widetilde{z}_{1}} \frac{1-\widetilde{z}_{2}}{1+\widetilde{z}_{2}}}
$$

Thus, we arrive at (7.10)-(7.11).

\section{REFERENCES}

[1] W.N. Anderson, Shorted operators, SIAM J. Appl. Math. 20 (1971), 520-525.

[2] W.N. Anderson and R.J. Duffin, Series and parallel addition of matrices, J. Math. Anal. Appl. 26 (1969), 576-594.

[3] W.N. Anderson and G.E. Trapp, Shorted operators, II, SIAM J. Appl. Math. 28 (1975), 60-71.

[4] W.N. Anderson, T.D. Morlye, and G.E. Trapp, Characterizations of parallel subtraction, Proc. Nat. Acad. Sci. USA, 76 (1975), No.8, 3599-3601.

[5] T. Ando, Lebesgue type decomposition of positive operators. Acta Sci.Math. (Szeged) 38 (1976), 253-260.

[6] T. Ando, Topics on operator inequalities, Lecture Notes, Hokkaido University, Sapporo, Japan, 1978.

[7] T. Ando and K. Nishio, Positive self-adjoint extensions of positive symmetric Operators. Tohóku Math. J. 22 (1970), 65-75.

[8] J. Antezana, G. Corach, D. Stojanoff, Bilateral shorted operators and parallel sums, Linear Algebra and Application 414 (2006), 570-588.

[9] R. Arens, Operational calculus of linear relations, Pacific J. Math. 11 (1961), 9-23. 
[10] Yu.M. Arlinskiu, On the theory of operator means. Ukr. Mat. Zh. 42 (1990), No.6, 723-730 (in Russian). English translation in Ukr. Math. Journ. 42 (1990), No.6, 639-645.

[11] Yu.M. Arlinskiĭ, Extremal extensions of sectorial linear relations. Matematychnii Studii 7 (1997), No.1, 81-96.

[12] Yu. Arlinskiı̌, S. Belyi, and E. Tsekanovskiǔ, Conservative realizations of Herglotz-Nevanlinna functions, Operator Theory: Advances and Applications 217. Basel: Birkhauser, xviii, 528 p.(2011).

[13] Yu. Arlinskiı̌ and S. Hassi, Holomorphic operator valued functions generated by passive selfadjoint systems, in Interpolation and Realization Theory with Applications to Control Theory, Operator Theory: Advances and Appl. 272 (2019), 35-76.

[14] Yu.M. Arlinskiü, S. Hassi, Z. Sebestyén, and H.S.V. de Snoo, On the class of extremal extensions of a nonnegative operator, Oper. Theory: Adv. Appl. 127, 41-81 (2001).

[15] Gr. Arsene and A. Gheondea, Completing matrix contractions, J. Operator Theory 7 (1982), 179-189.

[16] T.Ya. Azizov, A. Dijksma, and G. Wanjala, Compressions of maximal dissipative and self-adjoint linear relations and of dilations. Linear Algebra Appl. 439 (2013), 771-792.

[17] Ju. Behrndt, S. Hassi, H. de Snoo, and R. Wietsma, Monotone convergence theorems for semi-bounded operators and forms with applications, Proc. Royal Soc. of Edinburgh 140 A (2010), 927-951.

[18] E.A. Coddington and H.S.V. de Snoo, Positive selfadjoint extensions of positive symmetric subspaces, Math. Z. 159 (1978), 203-214.

[19] M. Contino, A. Maestripieri, and S. Marcantognini, Schur complements of selfadjoint Krĕn space operators, Linear Algebra and its Applications, 581 (2019), 214-246.

[20] Ch. Davis, W.M. Kahan, and H.F. Weinberger, Norm preserving dilations and their applications to optimal error bounds, SIAM J. Numer. Anal. 19 (1982), 445-469.

[21] V.A. Derkach and M.M. Malamud, Generalized resolvents and the boundary value problems for Hermitian operators with gaps, J. Funct. Anal. 95 (1991), No. 1, 1-95.

[22] V. Derkach, S. Hassi, M.M. Malamud, and H.S.V. de Snoo, Boundary relations and their Weyl families, Trans. Amer. Math. Soc. 358 (2006), No. 12, 5351-5400

[23] R.G. Douglas, On majorization, factorization and range inclusion of operators in Hilbert space, Proc. Amer. Math. Soc. 17 (1966), 413-416.

[24] W.G. Faris, Selfadjoint operators, Lecture Notes in Mathematics, 433 (1975).

[25] B. Farkas and M. Matolsci, Commutation properties of the form sum of positive, symmetric operators, Acta Sci.Math. (Szeged) 67 (2001), 777-790.

[26] P.A. Fillmore and J.P. Williams, On operator ranges. Advances in Math. 7 (1971), 254-281.

[27] J. Fridrich, M. Güntner, and L. Klotz, A generalized Schur complement for nonnegative operators on linear spaces, Banach J. Math. Anal 12 (2018), No.3, 617-633.

[28] S. Hassi, M. Malamud, and H.S.V. de Snoo, On Kreı̆n's extension theory of nonnegative operators, Math. Nach. 274-275 (2004), 40-73.

[29] S. Hassi, A. Sandovici, H.S.V. de Snoo, and H. Winkler, Form sums of nonnegative selfadjoint operators, Acta Math. Hungar. 111 (1-2) (2006), 81-105.

[30] S. Hassi, A. Sandovici, H.S.V. de Snoo, and H. Winkler, A general factorization approach to the extension theory of nonnegative operators and relations, J. Operator Theory 58 (2007), 351-386.

[31] S. Hassi, Z. Sebestyen, and H.S.V. de Snoo, Lebesgue type decompositions for nonnegative forms, J. Funct. Anal. 257 (2009), No.12, 3858-3894.

[32] S. Hassi, Z. Sebestyen, and H.S.V. de Snoo, Domain and range descriptions for adjoint relations, and parallel sums and differences of forms. Recent advances in operator theory in Hilbert and Krein spaces, 211-227, Oper. Theory Adv. Appl. 198, Birkhauser Verlag, Basel, 2010.

[33] T. Kato, Perturbation theory for linear operators, Springer-Verlag, Berlin, Heidelberg, 1995.

[34] M.G. Kreln, Theory of selfadjoint extensions of semibounded operators and its applications. I, Mat. Sb. 20 (1947), No.3, 431-498. (in Russian)

[35] M.G. Krĕn and I.E. Ovcharenko, On $Q$-functions and sc-extensions of nondensely defined Hermitian contractions, Sibirsk. Mat. Zh. 18 (1977), No.5, 1032-1056 (in Russian). English translation in Siberian Math. Journ. 18 (1977), No.5, 728-746.

[36] F. Kubo and T. Ando, Means of positive linear operators. Math. Ann. 246 (1980), No.3, 205-224. 
[37] A. Maestripieri and F.M. Peria, Schur complements in Kre ̌n spaces, Integral Equations Operator Theorey, 59 (2007), No.2, 207-221.

[38] S.K. Mitra, P. Bhimasankaram, S.B. Malik, Matrix partial orders, shorted operators and applications, Series in Algebra 10. Hackensack, NJ: World Scientific, 2010.

[39] T.D. Morley, Shorts of block operators and infinite networks-A note of the shorted operator: II, Circuits Systems Signal Process 9 (1990), No.2, 161-170

[40] K. Nishio and T. Ando, Characterizations of operators derived from network connections, J. Math. Anal. Appl. 53 (1976), 539-549.

[41] M.A. Nudelman, A generalization of Stenger's lemma to maximal dissipative operators, Integral Equations Operator Theory 70 (2011), 301-305.

[42] E.L. Pekarev, Shorts of operators and some extremal problems, Acta Sci. Math. (Szeged) 56 (1992), 147-163.

[43] E.L. Pekarev, A note on characterizations of the shorted operators, Electronic Journal of Linear Algebra 27 (2014), 155-161.

[44] E.L. Pekarev and Yu.L. Shmulyan, Parallel addition and parallel subtraction of operators. Izv. AN SSSR 40 (1976), 366-387 (1976) (in Russian). English translation in Math. USSR Izv. 10, 289-337.

[45] W. Pusz and S.L. Woronowics, Functional calculus for sesquilinear forms and purification map, Rep. Math. Phys., 8 (1975), No.2, 159-170.

[46] F.S. Rofe-Beketov, Numerical range of linear relation and maximal linear relations, Functions Theory, Functional Anal. and their Appl. 44 (1985), 103-111 (in Russian). English translation in Journal of Math. Sci. 48 (1990), No.3, 329-336.

[47] Yu.L. Shmul'yan, Hellinger's operator integral, Mat. Sb. 49 (1959), No.4, 381-430 (in Russian).

[48] Yu.L. Shmul'yan and R.N. Yanovskaya, Blocks of a contractive operator matrix, Izv. Vuzov, Mat. 7 (1981), 72-75 (in Russian). English translation in Sov. Math. 25 (1981), No. 7, 82-86.

[49] B. Simon, A canonical decomposition for quadratic forms with applications to monotone convergence theorems, J. Funct. Analysis 28 (1978), 377-385.

[50] W. Stenger, On the projection of a selfadjoint operator, Bull. Amer.Math.Soc. 74 (1968), 369-372.

[51] Z. Tarcsay, Lebesgue type decomposition of positive operators. Positivity 17 (2013), No.3, 803-817.

[52] Z. Tarcsay, On the parallel sum of positive operators, forms and functionals, Acta Math. Hungar. 147 (2015), No.2, 408-426.

[53] F. Zhang, The Schur complement and its applications. Springer, 2005.

Volodymyr Dahl East Ukrainian National University, pr. Central 59-A, Severodonetsk, 93400, UKRAINE

E-mail address: yury.arlinskii@gmail.com 